\title{
Normalized doubly coprime factorizations for infinite-dimensional linear systems
}

\author{
Ruth F. Curtain and Mark R. Opmeer \\ Math. Control Signals Systems 18 (2006), no. 1, 1-31
}

\begin{abstract}
We obtain explicit formulas for normalized doubly coprime factorizations of the transfer functions of the following class of linear systems: the input and output operators are vector-valued, but bounded, and the system is input and output stabilizable. Moreover, we give explicit formulas for the Bezout factors. Using a reciprocal approach we extend our results to a larger class where the input and output operators are allowed to be unbounded. This class is much larger than the class of well-posed linear systems.
\end{abstract}

\section{Introduction}

The representation of a transfer function as a coprime factorization has proven to be a powerful tool in systems theory. An important result for rational transfer functions is the Youla-Bongiorno parameterization of all stabilizing controllers in terms of doubly coprime factorizations of the rational transfer function and its Bezout factors. If $\Sigma(A, B, C, D)$ is a stabilizable and detectable realization of the rational transfer function, then explicit formulas for a doubly coprime factorization are known (see Nett et al. [24] and Francis [11]). Another important result in finite-dimensional systems theory is that of robustly stabilizing controllers with respect to factor perturbations. This relies on the existence of normalized left- or right-coprime factorizations (see Glover and McFarlane [13] and Georgiou and Smith [12]). Meyer and Franklin [22] obtained formulas for normalized coprime factorizations for any stabilizable and detectable realization $\Sigma(A, B, C, D)$ of a 
rational transfer function. These formulas show that coprime factorization is intimately connected to Riccati equation theory and we formulate these well-known results in a slightly different way.

We recall that $\Sigma(A, B, C, D)$ is stabilizable if and only if it satisfies the finite cost condition: for each $z_{0}$ there exists a $u \in \mathbf{L}_{2}(0, \infty ; U)$ such that

$$
J\left(z_{0}, u\right):=\int_{0}^{\infty}\|y(t)\|^{2}+\|u(t)\|^{2} d t<\infty,
$$

where $\dot{z}(t)=A z(t)+B u(t) ; z(0)=z_{0} ; y(t)=C z(t)+D u(t)$.

Similarly, $\Sigma(A, B, C, D)$ is detectable if and only if its dual system $\Sigma\left(A^{*}, C^{*}, B^{*}, D^{*}\right)$ satisfies the finite cost condition. If $\Sigma(A, B, C, D)$ and $\Sigma\left(A^{*}, C^{*}, B^{*}, D^{*}\right)$ satisfy the finite cost condition, then there exist unique nonnegative solutions $Q, P$ of the control and filter Riccati equations, respectively:

$$
\begin{aligned}
& A^{*} Q+Q A+C C^{*}=\left(Q B+C^{*} D\right) S^{-1}\left(B^{*} Q+D^{*} C\right), \\
& A P+P A^{*}+B B^{*}=\left(P C^{*}+B D^{*}\right) R^{-1}\left(C P+D B^{*}\right),
\end{aligned}
$$

where $S:=I+D^{*} D, F:=-S^{-1}\left(D^{*} C+B^{*} Q\right), R:=I+D D^{*}$ and $L:=$ $-\left(P C^{*}+B D^{*}\right) R^{-1}$. Moreover, $A_{Q}:=A+B F$ and $A_{P}:=A+L C$ are Hurwitz. A normalized doubly coprime factorization: $\mathbf{G}=\mathbf{N M}^{-1}=\tilde{\mathbf{M}}^{-1} \tilde{\mathbf{N}}$ with

$$
\left[\begin{array}{cc}
\tilde{\mathbf{X}} & -\tilde{\mathbf{Y}} \\
-\tilde{\mathbf{N}} & \tilde{\mathbf{M}}
\end{array}\right]\left[\begin{array}{cc}
\mathbf{M} & \mathbf{Y} \\
\mathbf{N} & \mathbf{X}
\end{array}\right]=I
$$

is given by

$$
\begin{aligned}
& {\left[\begin{array}{cc}
\mathbf{M} & \mathbf{Y} \\
\mathbf{N} & \mathbf{X}
\end{array}\right]=\left[\begin{array}{cc}
S^{-1 / 2} & 0 \\
D S^{-1 / 2} & R^{-1 / 2}
\end{array}\right]+\left[\begin{array}{c}
F \\
(C+D F)
\end{array}\right]\left(s I-A_{Q}\right)^{-1}\left[B S^{-1 / 2},-L R^{-1 / 2}\right],} \\
& {\left[\begin{array}{cc}
\tilde{\mathbf{X}} & -\tilde{\mathbf{Y}} \\
-\tilde{\mathbf{N}} & \mathbf{\mathbf { M }}
\end{array}\right]=\left[\begin{array}{cc}
S^{-1 / 2} & 0 \\
R^{-1 / 2} D & R^{-1 / 2}
\end{array}\right]+\left[\begin{array}{c}
S^{1 / 2} F \\
R^{-1 / 2} C
\end{array}\right]\left(s I-A_{P}\right)^{-1}[-(B+L D), L] .}
\end{aligned}
$$

While any proper rational transfer function has a (normalized) doubly coprime factorization over the field of stable rational transfer functions, not all irrational transfer functions have coprime factorizations. In [16] Inouye showed that a matrix-valued transfer function has a coprime factorization over $\mathbf{H}_{\infty}$ if and only if it is input-output stabilizable (see also Smith [31]). 
These are only existence results and give no procedure for finding formulas for a coprime factorization. For control applications we are interested in explicit formulas for the coprime factorizations and the Bezout factors in terms of the system parameters $A, B, C, D$. Some steps in this direction have been made for special classes of exponentially stabilizable and detectable infinitedimensional systems. The finite-dimensional results sketched above generalize perfectly for exponentially stabilizable and detectable infinite-dimensional systems with bounded input and output operators (Curtain and Zwart [6, Chapters $7,9.4]$ ) and for exponentially stabilizable and detectable regular linear systems with unbounded input and output operators (Curtain, Weiss and Weiss [2]). However, there are many infinite-dimensional systems that are not exponentially stabilizable or detectable, but which do have coprime factorizations. In Curtain and Oostveen [4] and Oostveen [28] formulas for a doubly coprime factorization using solutions to Riccati equations are derived for a class with bounded input and output operators under the following assumptions: $A$ has no essential spectrum on the imaginary axis, $U$ and $Y$ are finite-dimensional, and the system and its dual satisfy the finite cost condition. Formulas for the Bezout factors were not obtained. A more abstract approach to this problem has been taken by Staffans in [33] and Mikkola in [23] where they relate the existence of coprime factorizations to abstract stabilizability and detectability notions. However, the price paid for this generality is the loss of explicit formulas in terms of the generating operators and the difficulty of verifying these abstract stabilizability and detectability conditions (which are a priori stronger than the finite cost condition for the system and its dual).

By contrast, our approach is simple and explicit. We first study the special case of state linear systems, for which the input and output operators are bounded. We show that the transfer function of a state linear system has a normalized doubly coprime factorization provided that the state linear system and its dual satisfy the finite cost condition. Moreover, we obtain explicit formulas for the normalized doubly coprime factorization, including the Bezout factors. Surprizingly, although our formulas for the coprime factors are consistent with the finite-dimensional ones given above, those for the Bezout factors are different. They contain an extra parameter $\sigma$ that must be chosen to lie between 1 and $r^{1 / 2}\left(P Q(I+P Q)^{-1}\right)$, where $r$ denotes the spectral radius and $P, Q$ are solutions of the Riccati equations. If $\Sigma$ is exponentially stabilizable and detectable, we can choose $\sigma=1$, but, in general, it is not known whether these candidate Bezout factors will be in $\mathbf{H}_{\infty}$. 
Using a reciprocal approach (introduced in Curtain [8]) we extend our results to transfer functions of integrated nodes. This class of systems contains the class of well-posed linear systems (the class studied by Staffans [33] and Mikkola [23]) and several systems that are not well-posed (for example the ones studied in Lasiecka and Triggiani [19], [20], [21]).

In the first 8 sections we study the special class of state linear systems, for which the input and output operators are bounded. In this case, the finite cost condition for the system and its dual is equivalent to input and output stabilizability. After reviewing some known results on state linear systems in Section 2, in Section 3 we prove some new results on energy preserving systems. We examine the control Riccati equation and the properties of the right factor system in Section 4. The key differences are that the Riccati equations need not have unique solutions and so there may be several right factor systems. In Section 5 we examine the right factorizations and by means of a counter example we show that they need not always be normalized. However, using the results on energy preserving systems from Section 3, we show that a sufficient condition for the normalization property to hold is to choose the smallest bounded nonnegative solution to the control Riccati equation in the formula for the right factor. A sufficient condition for all solutions to generate a normalized factorization is that $\sigma(A) \cap i \mathbb{R}$ has measure zero. To show that our candidate normalized right coprime factor is in fact coprime we use Nehari's theorem. In Section 6 we summarize some known results on Nehari's theorem from Curtain and Opmeer [10] and prove a crucial new one. In Section 7 we prove our main results: we give sufficient conditions for the existence of a normalized doubly coprime factorization and we give explicit formulas for the normalized doubly coprime factorization and for the Bezout factors. While the formulas for the normalized coprime factors are the same as the finite-dimensional ones, as mentioned above, the Bezout factors are not quite what one would expect. In Section 8 we use a reciprocal approach to extend the results in Sections 2-7 for state linear systems to the class of integrated nodes.

\section{State linear systems}

In this section we review known properties of state linear systems and prove some interesting new ones. Following the terminology in Curtain and Zwart [6] we call $\Sigma(A, B, C, D)$ a state linear system if $A$ is the infinitesimal gener- 
ator of a strongly continuous semigroup $T(\cdot)$ on a separable Hilbert space $Z$, $B \in \mathcal{L}(U, Z), C \in \mathcal{L}(Z, Y), D \in \mathcal{L}(U, Y)$ with $U, Y$ separable Hilbert spaces.

For an input $u \in \mathbf{L}_{2}^{\text {loc }}\left(\mathbb{R}^{+} ; U\right)$ and initial state $z_{0} \in Z$ the state $z(t) \in Z$ at time $t \in \mathbb{R}^{+}$is defined by

$$
z(t)=T(t) z_{0}+\int_{0}^{t} T(t-s) B u(s) d s .
$$

If $u$ is continuously differentiable and $z_{0} \in D(A)$, then $z$ as defined above is continuously differentiable and for each $t \in \mathbb{R}^{+} z(t) \in D(A)$ and satisfies

$$
\dot{z}(t)=A z(t)+B u(t), \quad z(0)=z_{0} .
$$

The output of the state linear system is defined by

$$
y(t)=C z(t)+D u(t) .
$$

The transfer function $\mathbf{G}$ of the state linear system $\Sigma(A, B, C, D)$ is defined by: $\mathbf{G}-D$ equals the Laplace transform of $C T(t) B$ (on some right half-plane).

Instead of using the concept of exponential stability, we work with the following stability concepts.

Definition 2.1 1. A state linear system is input stable if there exists a constant $\beta>0$ such that for all $u \in \mathbf{L}_{2}(0, \infty ; U)$

$\left\|\int_{0}^{\infty} T(t) B u(t) \mathrm{d} t\right\|^{2} \leq \beta \int_{0}^{\infty}\|u(t)\|^{2} \mathrm{~d} t ;$

2. A state linear system is output stable if there exists a constant $\gamma>0$ such that for all $z \in Z$

$\int_{0}^{\infty}\|C T(t) z\|^{2} \mathrm{~d} t \leq \gamma\|z\|^{2}$

3. A state linear system is input-output stable if the transfer function $\mathbf{G} \in \mathbf{H}_{\infty}(\mathcal{L}(U, Y))$.

4. A state linear system is a stable system if it is input, output and inputoutput stable.

We note that the above three stability concepts (input stable, output stable, input-output stable) are independent in the sense that any combination of two of them does not imply the third. 
Definition 2.2 The output map $\mathcal{C}: Z \rightarrow \mathbf{L}_{2}(0, \infty ; Y)$ of an output stable state linear system $\Sigma(A, B, C, D)$ is defined by $(\mathcal{C} z)(t):=C T(t) z$ and the observability Gramian by $L_{C}:=\mathcal{C}^{*} \mathcal{C}$.

The input map $\mathcal{B}: \mathbf{L}_{2}(0, \infty ; U) \rightarrow Z$ of an input stable state linear system is defined by $\mathcal{B} u:=\int_{0}^{\infty} T(s) B u(s) d s$ and the controllability Gramian by $L_{B}:=\mathcal{B B}^{*}$.

The properties input and output stability are related to the existence of solutions to Lyapunov equations (see Grabowski [14] and Hansen and Weiss $[15])$.

Lemma 2.3 The state linear system $\Sigma(A, B, C, D)$ is input stable if and only if the following controllability Lyapunov equation has a bounded nonnegative solution $L \in \mathcal{L}(Z)$ :

$$
A L z+L A^{*} z=-B B^{*} z \quad \text { for all } z \in D\left(A^{*}\right) .
$$

In this case, the controllability Gramian $L_{B}$ is the smallest bounded nonnegative solution of (3) and $L_{B}^{1 / 2} T(t)^{*} z \rightarrow 0$ as $t \rightarrow \infty$ for all $z \in Z$.

The state linear system $\Sigma(A, B, C, D)$ is output stable if and only if the following observability Lyapunov equation has a bounded nonnegative solution $L \in \mathcal{L}(Z):$

$$
A^{*} L z+L A z=-C^{*} C z \quad \text { for all } z \in D(A) .
$$

In this case, the observability Gramian $L_{C}$ is the smallest bounded nonnegative solution of (4) and $L_{C}^{1 / 2} T(t) z \rightarrow 0$ as $t \rightarrow \infty$ for all $z \in Z$.

The following new result shows that the Gramian is the only solution of the Lyapunov equation with the convergence property as in Lemma 2.3.

Lemma 2.4 Suppose the state linear system $\Sigma(A, B, C, D)$ is input stable and $L_{1}$ is a bounded nonnegative solution of the Lyapunov equation (3) such that $L_{1}^{1 / 2} T(t)^{*} z \rightarrow 0$ as $t \rightarrow \infty$ for all $z \in Z$. Then $L_{1}=L_{B}$, the controllability Gramian of the system.

Suppose the state linear system $\Sigma(A, B, C, D)$ is output stable and $L_{2}$ is a bounded nonnegative solution of the Lyapunov equation (4) such that $L_{2}^{1 / 2} T(t) z \rightarrow$ 0 as $t \rightarrow \infty$ for all $z \in Z$. Then $L_{2}=L_{C}$, the observability Gramian of the system. 
Proof We prove only the second statement, the first then follows from duality. Let $x \in D(A)$, define $z=T(s) x$, then we obtain from (4)

$$
\left\langle L_{2} T(s) x, A T(s) x\right\rangle+\left\langle A T(s) x, L_{2} T(s) x\right\rangle+\langle C T(s) x, C T(s) x\rangle=0 .
$$

We integrate from 0 to $t$ to obtain, using (1),

$$
\left\langle L_{2} T(t) x, T(t) x\right\rangle+\int_{0}^{t}\|C T(s) x\|^{2}=\left\langle L_{2} x, x\right\rangle .
$$

By continuity this holds for all $x \in Z$. Since, by assumption $L_{2}^{1 / 2} T(t) x \rightarrow 0$, and the second term on the left-hand side converges to $\|\mathcal{C} x\|^{2}$ which equals $\left\langle L_{C} x, x\right\rangle$ we have $\left\langle L_{2} x, x\right\rangle=\left\langle L_{C} x, x\right\rangle$. Since this holds for all $x \in Z$ we obtain $L_{2}=L_{C}$.

A related, but different, concept to that of a transfer function is that of the characteristic function which we denote by $\mathfrak{G}$.

$\mathfrak{G}(s):=D+C(s I-A)^{-1} B$ for all $s \in \rho(A)$. For $s$ in some right half-plane we have $\mathbf{G}=\mathfrak{G}$, but they may differ outside this region. For a counter example see Curtain and Zwart [6, Example 4.3.8] and for a more detailed discussion Zwart [35]. To examine the connection between the two we introduce the Laplace transforms of the input and output maps.

Definition 2.5 For an output stable state linear system we define $\widehat{\mathcal{C}}: Z \rightarrow$ $\mathbf{H}_{2}(Y)$ by $\widehat{\mathcal{C}} z:=\widehat{\mathcal{C} z}$.

For an input stable state linear system we define $\widehat{\mathcal{B}}$ for $u \in U, z \in Z, s \in \mathbb{C}_{0}^{+}$ by $\langle\widehat{\mathcal{B}}(s) u, z\rangle:=\left\langle u, \widehat{\mathcal{B}^{*} z}(\bar{s})\right\rangle$.

Rather surprizingly, under the assumption of either input or output stability, the characteristic function equals the transfer function in $\rho(A) \cap \mathbb{C}_{0}^{+}$.

Lemma 2.6 (Curtain and Opmeer [10, Lemma 3.4]).

1. If the state linear system $\Sigma(A, B, C, D)$ is output stable, then

$$
\begin{aligned}
& \mathbf{G}(s)=D+\widehat{\mathcal{C}}(s) B \quad \forall s \in \mathbb{C}_{0}^{+} \\
& \mathbf{G}(s)=D+C(s I-A)^{-1} B=\mathfrak{G}(s) \quad \forall s \in \mathbb{C}_{0}^{+} \cap \rho(A) .
\end{aligned}
$$


2. If the state linear system $\Sigma(A, B, C, D)$ is input stable, then (6) holds and

$$
\mathbf{G}(s)=D+C \widehat{\mathcal{B}}(s) \quad \forall s \in \mathbb{C}_{0}^{+} .
$$

The following result on boundary functions was proven in [10, Lemma 3.5].

Lemma 2.7 Let $\Sigma(A, B, C, D)$ be input or output stable and assume that either $\sigma(A) \cap i \mathbb{R}$ has measure zero or $U$ and $Y$ are finite-dimensional. Then there exists an almost everywhere defined function $\mathbf{G}_{0}: i \mathbb{R} \rightarrow \mathcal{L}(U, Y)$ such that for almost all $\omega \in \mathbb{R}$ and all nontangential paths we have

$$
\mathbf{G}_{0}(i \omega)=\lim _{s \rightarrow i \omega} \mathbf{G}(s) .
$$

If $i \omega \in \rho(A)$ then $\mathbf{G}_{0}(i \omega)=\mathfrak{G}(i \omega)$.

\section{$3 \quad$ Energy preserving systems}

In this section we prove some properties of energy-preserving state linear systems.

Definition 3.1 A state linear system is called $L$-energy-preserving if there exists an $L=L^{*} \in \mathcal{L}(X)$ such that for any input signal $u \in \mathbf{L}_{2}^{\text {loc }}\left(\mathbb{R}^{+} ; U\right)$, any initial state $z_{0} \in Z$ and any time $T \in \mathbb{R}^{+}$we have

$$
\langle L z(T), z(T)\rangle+\int_{0}^{T}\|y(t)\|^{2} d t=\left\langle L z_{0}, z_{0}\right\rangle+\int_{0}^{T}\|u(t)\|^{2} d t .
$$

The following lemma is rather obvious, but important.

Lemma 3.2 Any L-energy preserving system with $L$ nonnegative is output stable and input-output stable.

The next theorem gives algebraic conditions for a system to be energypreserving.

Theorem 3.3 A state linear system $\Sigma(A, B, C, D)$ is L-energy-preserving if

$$
A^{*} L+L A+C^{*} C=0 \text { on } D(A), \quad D^{*} D=I, \quad B^{*} L=-D^{*} C .
$$


Proof Let $u \in \mathbf{L}_{2}^{\text {loc }}\left(\mathbb{R}^{+} ; U\right)$ be continuously differentiable. Then for $z_{0} \in$ $D(A)$, the state $z$ is continuously differentiable, $z(t) \in D(A)$ and it satisfies $\dot{z}(t)=A z(t)+B u(t)$. We obtain

$$
\begin{aligned}
& \frac{d}{d t}\langle L z(t), z(t)\rangle=\langle L[A z(t)+B u(t)], z(t)\rangle+\langle z(t), L[A z(t)+B u(t)]\rangle . \\
& =\left\langle\left[L A+A^{*} L\right] z(t), z(t)\right\rangle+\langle B u(t), L z(t)\rangle+\langle L z(t), B u(t)\rangle .
\end{aligned}
$$

Integrating from zero to $T$ we obtain

$$
\begin{aligned}
& \langle L z(T), z(T)\rangle-\left\langle L z_{0}, z_{0}\right\rangle \\
& =\int_{0}^{T}\left\langle\left[L A+A^{*} L\right] z(t), z(t)\right\rangle+\left\langle u(t), B^{*} L z(t)\right\rangle+\left\langle B^{*} L z(t), u(t)\right\rangle d t \\
& =-\int_{0}^{T}\left\langle C^{*} C z(t), z(t)\right\rangle-\left\langle D^{*} C z(t), u(t)\right\rangle-\left\langle u(t), D^{*} C z(t)\right\rangle d t
\end{aligned}
$$

On the other hand, we have

$$
\begin{gathered}
\int_{0}^{T}\langle y(t), y(t)\rangle d t=\int_{0}^{T}\langle C z(t)+D u(t), C z(t)+D u(t)\rangle d t \\
=\int_{0}^{T}\left\langle C^{*} C z(t), z(t)\right\rangle+\left\langle D^{*} C z(t), u(t)\right\rangle+\left\langle u(t), D^{*} C z(t)\right\rangle+\left\langle D^{*} D u(t), u(t)\right\rangle d t .
\end{gathered}
$$

We now see that (9) implies (8) provided that $u$ is continuously differentiable for all $z_{0} \in Z$, since $D(A)$ is dense in $Z$. The general case follows by approximating a locally square integrable $u$ by continuously differentiable functions.

The following corollary is proven in the same way as Theorem 3.3.

Corollary 3.4 If (9) holds, then for input signals $u^{1}, u^{2} \in \mathbf{L}_{2}^{\operatorname{loc}}\left(\mathbb{R}^{+} ; U\right)$, initial states $z_{0}^{1}, z_{0}^{2} \in Z$ and any time $T \in \mathbb{R}^{+}$we have

$$
\left\langle L z^{1}(T), z^{2}(T)\right\rangle+\int_{0}^{T}\left\langle y^{1}(t), y^{2}(t)\right\rangle d t=\left\langle L z_{0}^{1}, z_{0}^{2}\right\rangle+\int_{0}^{T}\left\langle u^{1}(t), u^{2}(t)\right\rangle d t .
$$

The following lemma is probably known, but we could not find a proof in the existing literature. 
Lemma 3.5 Let $y^{i}$ be the output of the state linear system $\Sigma(A, B, C, D)$ corresponding to the input $u^{i} \in \mathbf{L}_{2}\left(\mathbb{R}^{+} ; U\right)$ and $z_{0}^{i}=0$ for $i=1,2$. If for all $u^{1}, u^{2} \in \mathbf{L}_{2}\left(\mathbb{R}^{+} ; U\right)$ there holds

$$
\int_{0}^{\infty}\left\langle u^{1}(t), u^{2}(t)\right\rangle d t=\int_{0}^{\infty}\left\langle y^{1}(t), y^{2}(t)\right\rangle d t
$$

then the transfer function $\mathbf{G}$ has a boundary function and for almost all $\omega \in \mathbb{R}$ we have $\mathbf{G}(i \omega)^{*} \mathbf{G}(i \omega)=I$.

Proof The given equation with $u_{1}=u_{2}$ shows that the state linear system maps $\mathbf{L}_{2}\left(\mathbb{R}^{+} ; U\right)$ inputs into $\mathbf{L}_{2}\left(\mathbb{R}^{+} ; Y\right)$ outputs. It follows as in Weiss [34] that $\mathbf{G} \in \mathbf{H}_{\infty}(\mathcal{L}(U, Y))$. Hence $\mathbf{G}$ has an almost everywhere defined boundary function.

Let $u_{1}, u_{2} \in \mathbf{L}_{2}(\mathbb{R} ; U)$ with support bounded to the left. Suppose that $\tau$ is such that $u_{1}$ and $u_{2}$ are equal to zero on $(-\infty, \tau)$. If $\underline{u}_{i}(t):=u_{i}(t+$ $\tau)$, then $\underline{u}_{i}$ is equal to zero on $(-\infty, 0)$ and $\underline{u}_{i} \in \mathbf{L}_{2}\left(\mathbb{R}^{+} ; U\right)$. Let $y_{i}(t):=$ $\int_{-\infty}^{t} C T(t-s) B u^{i}(s) d s+D u^{i}(t)$ be the output corresponding to $u_{i}$ and define $\underline{y}_{i}(t):=y_{i}(t+\tau)$. Invoking the time-invariance property, $\underline{y}_{i}$ is the output corresponding to $\underline{u}_{i}$ and by causality $\underline{y}_{i}$ is equal to zero on $(-\infty, 0)$. Hence

$$
\int_{0}^{\infty}\left\langle\underline{u}^{1}(t), \underline{u}^{2}(t)\right\rangle d t=\int_{0}^{\infty}\left\langle\underline{y}^{1}(t), \underline{y}^{2}(t)\right\rangle d t
$$

and so

$$
\int_{-\infty}^{\infty}\left\langle u^{1}(t), u^{2}(t)\right\rangle d t=\int_{-\infty}^{\infty}\left\langle y^{1}(t), y^{2}(t)\right\rangle d t
$$

Taking Fourier transforms we obtain

$$
\int_{-\infty}^{\infty}\left\langle\hat{u}^{1}(i \omega), \hat{u}^{2}(i \omega)\right\rangle d \omega=\int_{-\infty}^{\infty}\left\langle\hat{y}^{1}(i \omega), \hat{y}^{2}(i \omega)\right\rangle d \omega .
$$

Now $\hat{y}(i \omega)=\mathbf{G}(i \omega) \underline{\hat{u}}(i \omega)$ and since $\underline{\hat{u}}(i \omega)=\mathrm{e}^{\tau i \omega} \hat{u}(i \omega)$ and $\underline{\hat{y}}(i \omega)=\mathrm{e}^{\tau i \omega} \hat{y}(i \omega)$, we obtain $\hat{y}(i \omega)=\mathbf{G}(i \omega) \hat{u}(i \omega)$. Substituting this in the above we obtain

$$
\int_{-\infty}^{\infty}\left\langle\hat{u}^{1}(i \omega), \hat{u}^{2}(i \omega)\right\rangle d \omega=\int_{-\infty}^{\infty}\left\langle\mathbf{G}(i \omega)^{*} \mathbf{G}(i \omega) \hat{u}^{1}(i \omega), \hat{u}^{2}(i \omega)\right\rangle d \omega
$$


and so

$$
\int_{-\infty}^{\infty}\left\langle\left(\mathbf{G}(i \omega)^{*} \mathbf{G}(i \omega)-I\right) \hat{u}^{1}(i \omega), \hat{u}^{2}(i \omega)\right\rangle d \omega=0 .
$$

This shows that $\left(\mathbf{G}(i \omega)^{*} \mathbf{G}(i \omega)-I\right) \hat{u}^{1}(i \omega)$ is orthogonal to a dense subset of $\mathbf{L}_{2}(i \mathbb{R} ; U)$ and hence equals zero. Let $f: \mathbb{R} \rightarrow \mathbb{C}$ be a function that has compact support and such that $\hat{f}(i \omega) \neq 0$ for almost all $\omega \in \mathbb{R}$ (for example the function equal to 1 on $[0,1]$ and zero elsewhere). Now let $u \in U$ and define $u_{1}(t)=f(t) u$. Since $\hat{f}(i \omega)$ is nonzero for almost all $\omega \in \mathbb{R}$ we have for almost all $\omega \in \mathbb{R}$

$$
\left(\mathbf{G}(i \omega)^{*} \mathbf{G}(i \omega)-I\right) u=0 .
$$

Since $u$ was arbitrary this proves that for almost all $\omega \in \mathbb{R}$ we have $\mathbf{G}(i \omega)^{*} \mathbf{G}(i \omega)=$ $I$.

We quote the following result from Curtain and Opmeer [10, Lemma 3.1].

Lemma 3.6 If $\Sigma(A, B, C, D)$ is output stable with observability Gramian $L_{C}$, then for all $u \in \mathbf{L}_{2}\left(\mathbb{R}^{+} ; U\right)$ with compact support

$$
L_{C}^{1 / 2} \int_{0}^{t} T(t-s) B u(s) d s \rightarrow 0 \text { as } t \rightarrow \infty .
$$

The following corollary is crucial in obtaining the normalization property of our candidate normalized coprime factor in Theorem 5.7.

Corollary 3.7 The transfer function of an output stable state linear system $\Sigma(A, B, C, D)$ for which the observability Gramian satisfies $B^{*} L_{C}=-D^{*} C$ and $D^{*} D=I$ holds is inner.

Proof First we note that since $L_{C}$ satisfies (4), Theorem 3.3 implies that $\Sigma(A, B, C, D)$ is energy preserving and by Lemma 3.2 it is input-output stable. Next in (10) substitute $u^{1}, u^{2} \in \mathbf{L}_{2}^{\text {loc }}\left(\mathbb{R}^{+} ; U\right)$ with compact support, $z_{0}^{1}=z_{0}^{2}=0$ and $L=L_{C}$. Letting $T \rightarrow \infty$ and using Lemma 3.6 we conclude that

$$
\int_{0}^{\infty}\left\langle y_{1}(t), y_{2}(t)\right\rangle d t=\int_{0}^{\infty}\left\langle u_{1}(t), u_{2}(t)\right\rangle d t .
$$

Let $u^{1}, u^{2} \in \mathbf{L}_{2}\left(\mathbb{R}^{+} ; U\right)$ and let $u_{n}^{1}, u_{n}^{2}$ be approximating sequences of functions with compact support. Since $\Sigma(A, B, C, D)$ is input-output stable 
$y_{n}^{i}, y^{i} \in \mathbf{L}_{2}\left(\mathbb{R}^{+} ; Y\right)$ and $y_{n}^{i} \rightarrow y^{i}$. From this it follows that (11) holds for all $u^{1}, u^{2} \in \mathbf{L}_{2}\left(\mathbb{R}^{+} ; U\right)$. Lemma 3.5 now gives the result.

We remark that the inner property need not hold for an arbitrary bounded nonnegative solution to the Lyapunov equation, since Lemma 3.6 only need hold for $L_{C}$, the smallest solution.

While input stability does not in general imply input-output stability, for $L$-energy preserving systems it does.

Lemma 3.8 An L-energy preserving system that is input stable is also inputoutput stable.

Proof Let $z_{0}=0$ and $u \in \mathbf{L}_{2}\left(\mathbb{R}^{+} ; U\right)$. From the input stability of the system we obtain the existence of a $\beta>0$ such that for all $t \geq 0$

$$
\|z(t)\|^{2}=\left\|\int_{0}^{t} T(t-s) B u(s) d s\right\|^{2} \leq \beta \int_{0}^{\infty}\|u(s)\|^{2} d s .
$$

This implies that for all $T \geq 0$ we obtain the estimate

$$
|\langle L z(T), z(T)\rangle| \leq\|L\| \beta \int_{0}^{\infty}\|u(s)\|^{2} d s
$$

and using (8) this implies that for all $T \geq 0$

$$
\begin{aligned}
& \int_{0}^{T}\|y(t)\|^{2} d t=\int_{0}^{T}\|u(t)\|^{2} d t-\langle L z(T), z(T)\rangle \\
& \leq \int_{0}^{T}\|u(t)\|^{2} d t+\|L\| \beta \int_{0}^{\infty}\|u(s)\|^{2} d s \leq(1+\|L\| \beta) \int_{0}^{\infty}\|u(s)\|^{2} d s .
\end{aligned}
$$

Hence $y \in \mathbf{L}_{2}\left(\mathbb{R}^{+} ; Y\right)$.

\section{Riccati equation theory}

There is a strong connection between coprime factorization and the linear quadratic regulator problem, which we review in this section. Consider the optimal control problem

$$
\min _{u \in \mathbf{L}_{2}(0, \infty ; U)} \int_{0}^{\infty}\|y(t)\|^{2}+\|u(t)\|^{2} d t .
$$


It is well-known (see Curtain and Zwart $[6$, Chapter 6$]$ ) that if for all initial states $z_{0} \in Z$ there exists an input $u \in \mathbf{L}_{2}\left(\mathbb{R}^{+} ; U\right)$ such that the output $y \in \mathbf{L}_{2}\left(\mathbb{R}^{+} ; Y\right)$, then for each $z_{0} \in Z$ there exists a unique $u^{\text {opt }} \in \mathbf{L}_{2}\left(\mathbb{R}^{+} ; U\right)$ for which the minimum is attained and there exists a bounded nonnegative operator $Q^{\mathrm{opt}}$ such that the minimal cost is given by $\left\langle Q^{\mathrm{opt}} z_{0}, z_{0}\right\rangle$. The optimal input can be given by a state feedback: $u^{\mathrm{opt}}(t)=F^{\mathrm{opt}} z^{\mathrm{opt}}(t)$, where $F^{\mathrm{opt}}:=$ $-S^{-1}\left(D^{*} C+B^{*} Q^{\mathrm{opt}}\right), S:=I+D^{*} D$.

$Q^{\text {opt }}$ is the smallest bounded nonnegative solution to the control algebraic Riccati equation on $D(A)$

$$
A^{*} Q+Q A+C^{*} C=\left(Q B+C^{*} D\right) S^{-1}\left(B^{*} Q+D^{*} C\right) .
$$

We introduce concepts of stabilizability that are refinements of the definitions introduced in Curtain and Oostveen [3].

Definition $4.1 \Sigma(A, B, C, D)$ is output stabilizable if there exists an $F \in$ $\mathcal{L}(Z, U)$ such that $\Sigma(A+B F, B,[C ; F], 0)$ is output stable.

$\Sigma(A, B, C, D)$ is input stabilizable if there exists an $L \in \mathcal{L}(Y, Z)$ such that $\Sigma(A+L C,[B, L], C, 0)$ is input stable.

Remark 4.2 It is easily seen that the finite cost condition holds if and only if the system is output stabilizable and the finite cost condition for the dual system holds if and only if the system is input stabilizable.

The following lemma relates stability and stabilizability.

Lemma 4.3 The state linear system $\Sigma(A, B, C, D)$ is a stable system if and only if it is input and output stabilizable and input-output stable.

Proof Without loss of generality we assume $D=0$. Let $F$ and $L$ be as in Definition 4.1 and denote $A_{F}:=A+B F$ and $A_{L}=A+L C$. Then

$$
C(s I-A)^{-1}=C\left(s I-A_{F}\right)^{-1}-C(s I-A)^{-1} B F\left(s I-A_{F}\right)^{-1}
$$

shows that $\Sigma(A, B, C, D)$ is output stable, and

$$
(s I-A)^{-1} B=\left(s I-A_{L}\right)^{-1} B-\left(s I-A_{L}\right)^{-1} L C(s I-A)^{-1} B
$$

shows that it is input stable. 
The following results are extensions of the results in Curtain and Oostveen [3]. In fact, they are special cases of analagous results for the very large class of well-posed linear systems in Mikkola [23], but [23] is not so accessible and our proofs are short.

Theorem 4.4 If the state linear system $\Sigma(A, B, C, D)$ is output stabilizable, then there exists a bounded nonnegative solution $Q$ of the control Riccati equation (12). Denote the closed-loop generator by $A_{Q}:=A+B F, F:=$ $-S^{-1}\left(D^{*} C+B^{*} Q\right), S:=I+D^{*} D$. Then the right factor system

$$
\Sigma\left(A_{Q}, B S^{-1 / 2},[C+D F ; F],[D ; I] S^{-1 / 2}\right)
$$

is output and input-output stable. If, in addition, $\Sigma(A, B, C, D)$ is input stabilizable, then the right factor system is a stable system.

If $\Sigma(A, B, C, D)$ is input stabilizable, then there exists a bounded nonnegative solution to the filter Riccati equation on $D\left(A^{*}\right)$

$$
A P+P A^{*}+B B^{*}=\left(P C^{*}+B D^{*}\right) R^{-1}\left(C P+D B^{*}\right),
$$

where $R:=I+D D^{*}$ and $L:=-\left(P C^{*}+B D^{*}\right) R^{-1}$. Moreover, the left factor system

$$
\Sigma\left(A_{P},[B+L D, L], R^{-1 / 2} C, R^{-1 / 2}[D, I]\right),
$$

where $A_{P}=A+L C$, is input and input-output stable. If, in addition, $\Sigma(A, B, C, D)$ is output stabilizable, then the left factor system is a stable system.

Proof The proof of the existence of a bounded nonnegative solution to $(12)$ is shown in $[6$, Theorem 6.2.4] for the case $D=0$. The case $D \neq 0$ can be reduced to the $D=0$ case by considering the system $\Sigma(A-$ $\left.B S^{-1} D^{*} C, B S^{-1 / 2}, R^{-1 / 2} C, 0\right)$ which has the same control Riccati equation. It is readily verified that the right factor system satisfies equations (9) with $\Sigma(A, B, C, D)$ replaced by (13) and $L$ replaced by $Q$. So it is $Q$-energy preserving and Corollary 3.2 shows that the right factor system is output and input-output stable.

Dual arguments apply to the filter Riccati equation.

The input stability of the closed-loop system can be proven as follows. Since $\Sigma(A, B, C, D)$ is input stabilizable, the filter Riccati equation has a bounded nonnegative solution $P$. As in Curtain and Zwart [6, Lemma 9.4.10] it can 
be shown that $(I+P Q)^{-1} P$ is a solution of the control Lyapunov equation of the right factor system. By Lemma 2.3 the closed-loop system is input stable.

The optimal right factor system (with $Q=Q^{\text {opt }}$ ) has a special property.

Theorem 4.5 Suppose that the state linear system $\Sigma(A, B, C, D)$ is output stabilizable, and let $Q^{\mathrm{opt}}$ denote the smallest bounded nonnegative solution to the control Riccati equation (12). Then the optimal right factor system

$$
\Sigma\left(A_{Q^{\mathrm{opt}}}, B S^{-1 / 2},\left[C+D F^{\mathrm{opt}} ; F^{\mathrm{opt}}\right],[D ; I] S^{-1 / 2}\right)
$$

corresponding to $Q^{\mathrm{opt}}$ has an inner transfer function.

Proof It is well-known that $Q^{\text {opt }}$ is the observability gramian of the system (16). Using this it is readily verified that the conditions of Corollary 3.7 are satisfied, which shows that (16) has an inner transfer function.

Dually, the optimal left factor system

$$
\Sigma\left(A_{P^{\mathrm{opt}}},\left[B+L^{\mathrm{opt}} D, L^{\mathrm{opt}}\right], R^{-1 / 2} C, R^{-1 / 2}[D, I]\right)
$$

corresponding to $P^{\text {opt }}$, the smallest bounded nonnegative solution to the filter Riccati equation (14), has a co-inner transfer function.

The following interesting properties about the spectrum of the closedloop generators $A_{Q}$ and $A_{P}$ on the right half-plane were shown in Curtain and Opmeer [10, Lemma 4.4].

Lemma 4.6 Suppose that the state linear system $\Sigma(A, B, C, D)$ is input and output stabilizable. Then there exist smallest bounded nonnegative solutions to the Riccati equations (12), (14), respectively, and for any pair of bounded nonnegative solutions $Q, P$ the closed-loop generators have following properties.

1. The closed-loop operators $A_{Q}=A-B S^{-1} D^{*} C-B S^{-1} B^{*} Q$ and $A_{P}=$ $A-B D R^{-1} C-P R^{-1} C^{*} C$ have the same spectrum and

$$
(I+P Q) A_{Q} z=A_{P}(I+P Q) z \text { for } z \in D(A) .
$$


2. The spectrum of the closed-loop generator $A_{Q}\left(A_{P}\right)$ in the right halfplane is contained in the spectrum of $A$ in the right half-plane. Moreover, $\sigma\left(A_{Q}\right) \cap i \mathbb{R}=\sigma\left(A_{P}\right) \cap i \mathbb{R} \subset \sigma(A) \cap i \mathbb{R}$.

From Lemma 4.6 and Lemma 2.3 we obtain the following.

Lemma 4.7 Suppose that the state linear system $\Sigma(A, B, C, D)$ is input and output stabilizable, $P^{\mathrm{opt}}$ is the smallest bounded nonnegative solution of the Riccati equation (14) and $Q$ is an arbitrary bounded nonnegative solution of the Riccati equation (12). Then for all $z \in Z$ we have $\left(P^{\mathrm{opt}}\right)^{1 / 2} T_{P \text { opt }}^{*}(t) z \rightarrow 0$ and $\left(P^{\text {opt }}\right)^{1 / 2} T_{Q}^{*}(t) z \rightarrow 0$ as $t \rightarrow \infty$.

Proof That $\left(P^{\text {opt }}\right)^{1 / 2} T_{P \text { opt }}^{*}(t) z \rightarrow 0$ follows from the fact that $P^{\text {opt }}$ is the controllability Gramian of the system (17) and Lemma 2.3. Since by Lemma 4.6 we have $T_{P \text { opt }}^{*}(t)=\left(I+Q P^{\mathrm{opt}}\right)^{-1} T_{Q}^{*}(t)\left(I+Q P^{\mathrm{opt}}\right)$ we obtain from this that $\left(P^{\mathrm{opt}}\right)^{1 / 2}\left(I+Q P^{\mathrm{opt}}\right)^{-1} T_{Q}^{*}(t) z \rightarrow 0$ for all $z \in Z$. Since $\left(P^{\text {opt }}\right)^{1 / 2}\left(I+Q P^{\text {opt }}\right)^{-1}=\left(I+\left(P^{\text {opt }}\right)^{1 / 2} Q\left(P^{\text {opt }}\right)^{1 / 2}\right)^{-1}\left(P^{\text {opt }}\right)^{1 / 2}$ we obtain $\left(P^{\mathrm{opt}}\right)^{1 / 2} T_{Q}^{*}(t) z \rightarrow 0$.

As we already saw in the proof of Theorem 4.4 there is a connection between the Riccati equations of $\Sigma(A, B, C, D)$ and the Lyapunov equations of the closed-loop system; we investigate this further.

Theorem 4.8 Suppose that the state linear system $\Sigma(A, B, C, D)$ is input and output stabilizable and $Q, P$ are arbitrary bounded nonnegative solutions of the Riccati equations (12), (14), respectively. Then the controllability and observability Lyapunov equations of the right factor system (13) have solutions $L_{1}=(I+P Q)^{-1} P$ and $L_{2}=Q$, respectively. Moreover, $r^{1 / 2}\left(L_{B} L_{C}\right) \leq r^{1 / 2}\left(L_{1} L_{2}\right)<1$.

Proof Note that the control Riccati equation can be reformulated as the observability Lyapunov equation of the right factor system

$$
A_{Q}^{*} Q+Q A_{Q}+Q B S^{-1} B^{*} Q+(C+D F)^{*}(C+D F)+F^{*} F=0 .
$$

This show that $L_{2}=Q$ is a solution of this Lyapunov equation. In the proof of Theorem 4.5 we pointed out that the observability Lyapunov equation for the right factor system is precisely (12). That $L_{1}=P(I+P Q)^{-1}$ is a solution to the controllability Lyapunov equation can be verified algebraically as in 
[6, Theorem 9.4.10]. Next note that for a bounded nonnegative operator $X$ we have

$$
(I+X)^{-1} X<I \Longleftrightarrow X(I+X)<(I+X)^{2} \Longleftrightarrow 0<I+X,
$$

and the latter is true, since $X \geq 0$. Applying this to $X=Q^{1 / 2} P Q^{1 / 2}$ we obtain

$$
I>\left(I+Q^{1 / 2} P Q^{1 / 2}\right)^{-1} Q^{1 / 2} P Q^{1 / 2}=Q^{1 / 2}(I+P Q)^{-1} P Q^{1 / 2} .
$$

Thus

$$
r\left(L_{1} L_{2}\right)=r\left((I+P Q)^{-1} P Q\right)=r\left(Q^{1 / 2}(I+P Q)^{-1} P Q^{1 / 2}\right)<1 .
$$

But we know that $L_{C}, L_{B}$ are the smallest solutions to the Lyapunov equations and so

$$
\begin{aligned}
& r\left(L_{B} L_{C}\right)=r\left(L_{C}^{1 / 2} L_{B} L_{C}^{1 / 2}\right) \leq r\left(L_{C}^{1 / 2} L_{1} L_{C}^{1 / 2}\right)=r\left(L_{1}^{1 / 2} L_{C} L_{1}^{1 / 2}\right) \\
& \leq r\left(L_{1}^{1 / 2} L_{2} L_{1}^{1 / 2}\right)=r\left(L_{1} L_{2}\right)<1 .
\end{aligned}
$$

The next lemma gives an explicit formula for the controllability Gramian of the right factor system.

Lemma 4.9 Suppose that the state linear system $\Sigma(A, B, C, D)$ is input and output stabilizable, $P^{\mathrm{opt}}$ is the smallest bounded nonnegative solution of the filter Riccati equation (14) and $Q$ is an arbitrary bounded nonnegative solution of the control Riccati equation (12). Then $L_{1}:=P^{\mathrm{opt}}\left(I+Q P^{\mathrm{opt}}\right)^{-1}=L_{B}$, the controllability Gramian of right factor system (13).

Proof According to Kato [17, Lemma V.3.43 page 284] we have the following representation for the square root of a bounded nonnegative operator $T$ :

$$
T^{1 / 2} z=\frac{1}{\pi} \int_{0}^{\infty} \lambda^{-1 / 2}(T+\lambda)^{-1} T z d \lambda
$$

and we have the following resolvent estimate [17, equation (V.3.38) page 279]

$$
\left\|(T+\lambda)^{-1}\right\| \leq \frac{1}{\lambda}
$$


for $\lambda>0$. Applying this with $L_{1}$ and $P^{\text {opt }}$ we obtain

$$
L_{1}^{1 / 2} z-\left(P^{\mathrm{opt}}\right)^{1 / 2} z=\frac{1}{\pi} \int_{0}^{\infty} \lambda^{-1 / 2}\left[\left(L_{1}+\lambda\right)^{-1} L_{1}-\left(P^{\mathrm{opt}}+\lambda\right)^{-1} P^{\mathrm{opt}}\right] z d \lambda
$$

and some rewriting of the integrand gives

$$
L_{1}^{1 / 2} z-\left(P^{\mathrm{opt}}\right)^{1 / 2} z=\frac{1}{\pi} \int_{0}^{\infty} \lambda^{1 / 2}\left(L_{1}+\lambda\right)^{-1} L_{1} Q\left(P^{\mathrm{opt}}+\lambda\right)^{-1} P^{\mathrm{opt}} z d \lambda .
$$

Using the above resolvent estimate we obtain

$$
\left\|\lambda^{1 / 2}\left(L_{1}+\lambda\right)^{-1} L_{1} Q\left(P^{\mathrm{opt}}+\lambda\right)^{-1} P^{\mathrm{opt}} z\right\| \leq \lambda^{-3 / 2}\left\|L_{1}\right\|\|Q\|\left\|P^{\mathrm{opt}} z\right\|
$$

and so

$$
\left\|\int_{1}^{\infty} \lambda^{1 / 2}\left(L_{1}+\lambda\right)^{-1} L_{1} Q\left(P^{\mathrm{opt}}+\lambda\right)^{-1} P^{\mathrm{opt}} z d \lambda\right\| \leq 2\left\|L_{1}\right\|\|Q\|\left\|P^{\mathrm{opt}} z\right\| .
$$

Since $\left(L_{1}+\lambda\right)^{-1} L_{1}=I-\lambda\left(L_{1}+\lambda\right)^{-1}$ we obtain from the above resolvent estimate $\left\|\left(L_{1}+\lambda\right)^{-1} L_{1}\right\| \leq 2$ and so

$$
\left\|\lambda^{1 / 2}\left(L_{1}+\lambda\right)^{-1} L_{1} Q\left(P^{\mathrm{opt}}+\lambda\right)^{-1} P^{\mathrm{opt}} z\right\| \leq 2 \lambda^{-1 / 2}\|Q\|\left\|P^{\mathrm{opt}} z\right\|,
$$

which gives

$$
\left\|\int_{0}^{1} \lambda^{1 / 2}\left(L_{1}+\lambda\right)^{-1} L_{1} Q\left(P^{\mathrm{opt}}+\lambda\right)^{-1} P^{\mathrm{opt}} z d \lambda\right\| \leq 4\|Q\|\left\|P^{\mathrm{opt}} z\right\| .
$$

Combining the above two estimates we obtain

$$
\left\|\int_{0}^{\infty} \lambda^{1 / 2}\left(L_{1}+\lambda\right)^{-1} L_{1} Q\left(P^{\mathrm{opt}}+\lambda\right)^{-1} P^{\mathrm{opt}} z d \lambda\right\| \leq 2\left(2+\left\|L_{1}\right\|\right)\|Q\|\left\|P^{\mathrm{opt}} z\right\|
$$

and so

$$
\left\|L_{1}^{1 / 2} z-\left(P^{\mathrm{opt}}\right)^{1 / 2} z\right\| \leq \frac{2}{\pi}\left(2+\left\|L_{1}\right\|\right)\|Q\|\left\|P^{\mathrm{opt}} z\right\|,
$$

which gives

$$
\left\|L_{1}^{1 / 2} z\right\| \leq\left\|\left(P^{\mathrm{opt}}\right)^{1 / 2} z\right\|+\frac{2}{\pi}\left(2+\left\|L_{1}\right\|\right)\|Q\|\left\|P^{\mathrm{opt}} z\right\| .
$$

With $z=T_{Q}^{*}(t) x$ and using Lemma 4.7 we obtain $L_{1}^{1 / 2} T_{Q}^{*}(t) x \rightarrow 0$ for all $x \in Z$. By Lemma 2.4 we obtain that $L_{1}$ is the Gramian. 


\section{Normalized Factorizations}

In this section we prove normalization and other properties of the transfer function $\left[\mathbf{N}^{Q} ; \mathbf{M}^{Q}\right]$ of the right factor (13) and of the transfer function $\left[\mathbf{N}^{\text {opt }} ; \mathbf{M}^{\mathrm{opt}}\right]$ of the optimal right factor (16) and of their respective characteristic functions $\left[\mathfrak{N}^{Q} ; \mathfrak{M}^{Q}\right]$ and $\left[\mathfrak{N}^{\text {opt }} ; \mathfrak{M}^{\text {opt}}\right]$. We recall some definitions.

First we define the precise concept of coprimeness we will work with.

Definition $5.1[\mathbf{N} ; \mathbf{M}] \in \mathbf{H}_{\infty}(\mathcal{L}(U, Y \oplus U))$ is right coprime if there exist $\tilde{\mathbf{X}}, \tilde{\mathbf{Y}}$ such that $[\tilde{\mathbf{X}}, \tilde{\mathbf{Y}}] \in \mathbf{H}_{\infty}(\mathcal{L}(U \oplus Y, U))$ and for all $s \in \mathbb{C}_{0}^{+}$there holds

$$
\tilde{\mathbf{X}}(s) \mathbf{M}(s)-\tilde{\mathbf{Y}}(s) \mathbf{N}(s)=I .
$$

Definition $5.2[\tilde{\mathbf{N}}, \tilde{\mathbf{M}}] \in \mathbf{H}_{\infty}(\mathcal{L}(Y \oplus U, Y))$ is left coprime over $\mathbb{C}_{0}^{+}$if there exist $\mathbf{X}, \mathbf{Y}$ such that $[\mathbf{X} ; \mathbf{Y}] \in \mathbf{H}_{\infty}(\mathcal{L}(Y, Y \oplus U))$ and for all $s \in \mathbb{C}_{0}^{+}$there holds

$$
\tilde{\mathbf{M}}(s) \mathbf{X}(s)-\tilde{\mathbf{N}}(s) \mathbf{Y}(s)=I .
$$

Of particular interest are normalized pairs.

Definition 5.3 We call $[\mathbf{N} ; \mathbf{M}] \in \mathbf{H}_{\infty}(\mathcal{L}(U, Y \oplus U))$ normalized if for almost all $\omega \in \mathbb{R}$ the following holds

$$
\mathbf{M}(i \omega)^{*} \mathbf{M}(i \omega)+\mathbf{N}(i \omega)^{*} \mathbf{N}(i \omega)=I .
$$

(i.e., $[\mathbf{N} ; \mathbf{M}]$ is inner).

We call $[\tilde{\mathbf{N}}, \tilde{\mathbf{M}}] \in \mathbf{H}_{\infty}(\mathcal{L}(Y \oplus U, Y))$ normalized if for almost all $\omega \in \mathbb{R}$ the following holds

$$
\tilde{\mathbf{M}}(i \omega) \tilde{\mathbf{M}}(i \omega)^{*}+\tilde{\mathbf{N}}(i \omega) \tilde{\mathbf{N}}(i \omega)^{*}=I .
$$

(i.e., $[\tilde{\mathbf{N}}, \tilde{\mathbf{M}}]$ is co-inner).

We now define coprime factorizations of a function $\mathbf{G}$.

Definition 5.4 The function $\mathbf{G}$ has a right-coprime factorization if there exist $[\mathbf{N} ; \mathbf{M}] \in \mathbf{H}_{\infty}(\mathcal{L}(U, Y \oplus U))$ that are right coprime, $\mathbf{M}$ has an inverse on some right half-plane and $\mathbf{G}(s)=\mathbf{N}(s) \mathbf{M}(s)^{-1}$ on some right half-plane. It has a left-coprime factorization if there exist $[\tilde{\mathbf{N}} ; \tilde{\mathbf{M}}] \in \mathbf{H}_{\infty}(\mathcal{L}(Y \oplus U, Y))$ that are left coprime, $\tilde{\mathbf{M}}$ has an inverse on some right half-plane and $\mathbf{G}(s)=$ $\tilde{\mathbf{M}}(s)^{-1} \tilde{\mathbf{N}}(s)$ on some right half-plane. 
Definition 5.5 A function has a doubly coprime factorization if it has a right-coprime factorization $[\mathbf{N} ; \mathbf{M}] \in \mathbf{H}_{\infty}(\mathcal{L}(Y \oplus U, U))$ with Bezout factor $[\tilde{\mathbf{X}}, \tilde{\mathbf{Y}}] \in \mathbf{H}_{\infty}(\mathcal{L}(U \oplus Y, U))$ and a left-coprime factorization $[\tilde{\mathbf{N}}, \tilde{\mathbf{M}}] \in$ $\mathbf{H}_{\infty}(\mathcal{L}(Y \oplus U, Y))$, with Bezout factor $[\mathbf{X} ; \mathbf{Y}] \in \mathbf{H}_{\infty}(\mathcal{L}(Y, Y \oplus U))$ such that

$$
\left[\begin{array}{cc}
\tilde{\mathbf{X}} & -\tilde{\mathbf{Y}} \\
-\tilde{\mathbf{N}} & \tilde{\mathbf{M}}
\end{array}\right]\left[\begin{array}{cc}
\mathbf{M} & \mathbf{Y} \\
\mathbf{N} & \mathbf{X}
\end{array}\right]=I=\left[\begin{array}{ll}
\mathbf{M} & \mathbf{Y} \\
\mathbf{N} & \mathbf{X}
\end{array}\right]\left[\begin{array}{cc}
\tilde{\mathbf{X}} & -\tilde{\mathbf{Y}} \\
-\tilde{\mathbf{N}} & \tilde{\mathbf{M}}
\end{array}\right]
$$

holds on $\mathbb{C}_{0}^{+}$.

We prove some properties of the transfer function $\left[\mathbf{N}^{Q} ; \mathbf{M}^{Q}\right]$ and of the characteristic function $\left[\mathfrak{N}^{Q} ; \mathfrak{M}^{Q}\right]$ of the right factor system (13).

Theorem 5.6 If $\Sigma(A, B, C, D)$ is output stabilizable with transfer function $\mathbf{G}$ and characteristic function $\mathfrak{G}$ then

1. $\left[\mathbf{N}^{Q} ; \mathbf{M}^{Q}\right] \in \mathbf{H}_{\infty}(\mathcal{L}(U, Y \oplus U))$.

2. $\mathfrak{M}^{Q}$ has an inverse on $\rho\left(A_{Q}\right) \cap \rho(A)$ which is the characteristic function of the state linear system

$$
\Sigma\left(A, B,-S^{1 / 2} F, S^{1 / 2}\right) .
$$

3. $\mathbf{M}^{Q}$ is invertible on some right half-plane and its inverse is the transfer function of the state linear system (24).

4. On $\rho(A) \cap \rho\left(A_{Q}\right)$ there holds $\mathfrak{G}=\mathfrak{N}^{Q} \mathfrak{M}^{Q^{-1}}$.

5. On $\mathbb{C}_{0}^{+} \cap \rho\left(A_{Q}\right)$, and hence on $\mathbb{C}_{0}^{+} \cap \rho(A)$, the transfer function $\left[\mathbf{N}^{Q} ; \mathbf{M}^{Q}\right]$ and the characteristic function $\left[\mathfrak{N}^{Q} ; \mathfrak{M}^{Q}\right]$ are equal.

6. On some right half-plane there holds $\mathbf{G}=\mathbf{N}^{Q} \mathbf{M}^{Q^{-1}}$.

7. If $\sigma(A) \cap i \mathbb{R}$ has measure zero, then the boundary function of the transfer function $\left[\mathbf{N}^{Q} ; \mathbf{M}^{Q}\right]$ and the characteristic function $\left[\mathfrak{N}^{Q} ; \mathfrak{M}^{Q}\right]$ are equal almost everywhere on $i \mathbb{R}$.

Proof 1. This is a consequence of Theorem 4.4.

2. This follows from an easy algebraic computation.

3. This follows from part 2. and the fact that the transfer function and characteristic function of a state linear system are equal on some right half-plane. 
4. This is an easy algebraic computation.

5. From Theorem 4.4 we obtain that the state linear system (13) is output stable and the result then follows from Lemma 2.6 part 1 and Lemma 4.6 part 2 .

6. This follows from parts 4 . and 5 .

7. This follows using Lemma 2.7.

We give sufficient conditions for the factorizations to be normalized.

Theorem 5.7 Suppose that the state linear system $\Sigma(A, B, C, D)$ with transfer function $\mathbf{G}$ is output stabilizable. Then

1. $\left[\mathbf{N}^{\mathrm{opt}} ; \mathbf{M}^{\mathrm{opt}}\right]$ is a normalized factorization of $\mathbf{G}$.

2. If the spectrum of $A$ on the imaginary axis has measure zero, then $\left[\mathbf{N}^{Q} ; \mathbf{M}^{Q}\right]$ is a normalized factorization of $\mathbf{G}$.

Proof 1. This follows from Theorem 4.5.

2. The formulas for $D \neq 0$ have been treated in detail in Curtain and Zwart [6, Theorem 7.3.11], so for simplicity we assume that $D=0$. We know from Lemma 4.6 part 2. and our assumption on $\sigma(A)$ that $\left(i \omega I-A_{Q}\right)^{-1}$ is bounded for almost all $\omega \in \mathbb{R}$ and direct calculation using (12) yields for almost all $\omega \in \mathbb{R}$

$$
\begin{aligned}
& \mathfrak{M}^{Q}(i \omega)^{*} \mathfrak{M}^{Q}(i \omega)+\mathfrak{N}^{Q}(i \omega)^{*} \mathfrak{N}^{Q}(i \omega)-I \\
&=\quad-B^{*} Q\left(i \omega I-A_{Q}\right)^{-1} B-B^{*}\left(-i \omega I-A_{Q}^{*}\right)^{-1} Q B+ \\
& B^{*}\left(-i \omega I-A_{Q}^{*}\right)^{-1}\left[C^{*} C+Q B B^{*} Q\right]\left(i \omega I-A_{Q}\right)^{-1} B \\
&=-B^{*} Q\left(i \omega I-A_{Q}\right)^{-1} B+B^{*}\left(i \omega I+A_{Q}^{*}\right)^{-1} Q B- \\
& B^{*}\left(i \omega I+A_{Q}^{*}\right)^{-1}\left(-i \omega I-A_{Q}^{*}\right) Q\left(i \omega I-A_{Q}\right)^{-1} B- \\
& B^{*}\left(i \omega I+A_{Q}^{*}\right)^{-1} Q\left(i \omega I-A_{Q}\right)\left(i \omega I-A_{Q}\right)^{-1} B \\
&= 0 .
\end{aligned}
$$

So $\left[\mathfrak{N}^{Q} ; \mathfrak{M}^{Q}\right]$ is normalized. The result now follows from Theorem 5.6 part 7.

That the condition in part 2. of Theorem 5.7 is not superfluous follows from the following example. 
Example 5.8 In Curtain and Sasane [7] the transfer function $\mathbf{G}(s)=\frac{1}{\sqrt{s^{2}+1}}$ is shown to have a realization $\Sigma\left(A, B, B^{*}, 0\right)$ on the state space $\ell_{2}(\mathbb{Z})$, where $A \in \mathcal{L}\left(\ell_{2}(\mathbb{Z})\right)$ and $B \in \ell_{2}(\mathbb{Z})$ are given by

$$
\begin{aligned}
A_{i, i+1} & =-1 / 2, A_{i+1, i}=1 / 2, A_{i, j}=0 \text { otherwise } ; \\
B_{0} & =1, B_{i}=0 \text { otherwise. }
\end{aligned}
$$

The spectrum of $A$ is purely continuous and equals $[-i, i]$. By inspection, the control and filter Riccati equations for $\Sigma\left(A, B, B^{*}, 0\right)$ have the solutions $P=Q=I$. So the system is input and output stabilizable and $\left[\mathbf{M}^{Q} ; \mathbf{N}^{Q}\right]$ is a right factor. In Curtain and Sasane [7] it is shown that $\mathbf{N}^{Q}(s)=\frac{1}{1+\sqrt{s^{2}+1}}$, $\mathbf{M}^{Q}(s)=I-\mathbf{N}^{Q}(s)$. Moreover, it is coprime with Bezout factors $\tilde{\mathbf{X}}=1=$ $-\tilde{\mathbf{Y}}$. However, a simple calculation shows that $\left[\mathbf{M}^{Q} ; \mathbf{N}^{Q}\right]$ is only normalized on $|\omega| \geq 1$, whereas for $|\omega| \leq 1$ there holds

$$
\mathbf{M}^{Q}(i \omega)^{*} \mathbf{M}^{Q}(i \omega)+\mathbf{N}^{Q}(i \omega)^{*} \mathbf{N}^{Q}(i \omega)=\frac{2-\omega^{2}}{2-\omega^{2}+2 \sqrt{1-\omega^{2}}}
$$

In [7] it is shown that $A_{Q}=A_{P}=A-B B^{*}$ generates a uniformly bounded (contraction) semigroup $T_{B}(t)$, but it is not strongly stable $\left(\sigma\left(A-B B^{*}\right)=\right.$ $\left.\sigma_{c}\left(A-B B^{*}\right)=[-i, i]\right)$. It is readily verified that $\left[\mathbf{M}^{Q} ; \mathbf{N}^{Q}\right]$ is continuous on the extended imaginary axis and so its Hankel operator is compact. Inspection of Theorem 6.2 shows that its Lyapunov equations must have a pair of solutions whose product is compact. Consequently, the Riccati equation solutions $P=Q=I$ are not unique. There must exist another solution pair $Q_{1}, P_{1}$ such that $P_{1} Q_{1}$ is compact and $Q_{1}, P_{1} \leq I$.

This example raises the interesting question: if $Q_{1}, Q_{2}$ are two solutions to the control Riccati equation and one of them generates a coprime factorization, does the other one?

Lemma 5.9 Suppose that $\Sigma(A, B, C, D)$ is input and output stabilizable with transfer function $\mathbf{G}$ and (12) has two self-adjoint nonnegative solutions $Q_{1}$ and $Q_{2}$. Define for $i=1,2\left[\mathbf{N}_{i} ; \mathbf{M}_{i}\right]$ as the transfer function of the state linear system (13) with $Q=Q_{i}$. Then $\left[\mathbf{N}_{1} ; \mathbf{M}_{1}\right]$ is a right-coprime factorization of $\mathbf{G}$ if and only if $\left[\mathbf{N}_{2} ; \mathbf{M}_{2}\right]$ is.

Proof For simplicity we take $D=0$. Define $\Delta:=Q_{1}-Q_{2}$. Then it is readily verified that

$$
\left[\mathbf{N}_{1} ; \mathbf{M}_{1}\right]=\left[\mathbf{N}_{2} ; \mathbf{M}_{2}\right] \mathbf{E}
$$


where $\mathbf{E}$ is the transfer function of the state linear system

$$
\Sigma\left(A_{Q_{1}}, B,-B^{*} \Delta, I\right) .
$$

$\mathbf{E}$ has an inverse which is the transfer function of the state linear system

$$
\Sigma\left(A_{Q_{2}}, B, B^{*} \Delta, I\right) .
$$

Since we have

$$
A_{Q_{1}}^{*} \Delta+\Delta A_{Q_{1}}+\Delta B B^{*} \Delta=0 \quad \text { on } D(A)
$$

it follows from Theorem 3.3 that (26) is $\Delta$-energy preserving and by Theorem 4.4 that (26) is input stable. Lemma 3.8 now shows that $\mathbf{E} \in \mathbf{H}_{\infty}(U)$. Similarly we have $\mathbf{E}^{-1} \in \mathbf{H}_{\infty}(U)$ since (27) is $-\Delta$-energy preserving.

Assume that $\left[\mathbf{N}_{1} ; \mathbf{M}_{1}\right]$ is right coprime, then there exist $\left[\tilde{\mathbf{X}}_{1}, \tilde{\mathbf{Y}}_{1}\right] \in \mathbf{H}_{\infty}(\mathcal{L}(U \oplus$ $Y, U)$ ) such that

$$
\tilde{\mathbf{X}}_{1} \mathbf{M}_{1}-\tilde{\mathbf{Y}}_{1} \mathbf{N}_{1}=I \text { on } \mathbb{C}_{0}^{+} .
$$

From (25) we deduce that

$$
\mathbf{E} \tilde{\mathbf{X}}_{1} \mathbf{M}_{2}-\mathbf{E} \tilde{\mathbf{Y}}_{1} \mathbf{N}_{2}=I,
$$

and since $\mathbf{E} \in \mathbf{H}_{\infty},\left[\tilde{\mathbf{X}}_{2}, \tilde{\mathbf{Y}}_{2}\right]=\mathbf{E}\left[\tilde{\mathbf{X}}_{1}, \tilde{\mathbf{Y}}_{1}\right]$ is a Bezout factor and $\left[\mathbf{N}_{2} ; \mathbf{M}_{2}\right]$ is right coprime.

This lemma shows that in our example all other solutions to the Riccati equation also provide a coprime factorization. Theorem 5.7 shows that the factor corresponding to the smallest one will also be normalized.

\section{The Nehari problem}

As mentioned in the introduction we obtain coprimeness from the solution of the suboptimal Nehari problem. The suboptimal Nehari problem is: for $\mathbf{G} \in \mathbf{L}_{\infty}(\mathcal{L}(U, Y))$ and a given $\sigma>\left\|H_{\mathbf{G}}\right\|$ ( $H_{\mathbf{G}}$ is the Hankel operator with symbol $\mathbf{G})$ find all $\mathbf{K}(-s) \in \mathbf{H}_{\infty}(\mathcal{L}(U, Y))$ such that

$$
\|\mathbf{G}+\mathbf{K}\|_{\infty} \leq \sigma .
$$


The vector-valued case was solved in Kheifets [18] (see also Peller [29]).

For our application we need a state space realization for a solution $\mathbf{K}(-s)$ in terms of a given state space realization for $\mathbf{G} \in \mathbf{H}_{\infty}(\mathcal{L}(U, Y))$. In [10] such an explicit solution was given under one of two assumptions: either $U, Y$ were finite-dimensional and $L_{1}=L_{B}, L_{2}=L_{C}$ or $\sigma(A) \cap i \mathbb{R}$ has measure zero. For our purposes one solution suffices and in this section we derive one such solution without assuming either of these conditions. We begin by recalling some basic definitions and results from [10].

Definition 6.1 For $\mathbf{G} \in \mathbf{L}_{\infty}((-i \infty, i \infty) ; \mathcal{L}(U, Y))$ we define the Hankel operator with symbol $\mathbf{G}$ as the operator $H_{\mathbf{G}}: \mathbf{H}_{2}(U) \rightarrow \mathbf{H}_{2}(Y)$ given by

$$
H_{\mathbf{G}} f=\Pi\left(\Lambda_{\mathbf{G}} f_{-}\right) \text {for } f \in \mathbf{H}_{2}(U),
$$

where $\Lambda_{\mathbf{G}}$ is the multiplication map on $\mathbf{L}_{2}((-i \infty, i \infty) ; U)$ induced by $\mathbf{G}, \Pi$ is the orthogonal projection from $\mathbf{L}_{2}((-i \infty, i \infty) ; U)$ onto $\mathbf{H}_{2}(U)$ and $f_{-}(s):=$ $f(-s)$.

Given $h \in \mathbf{L}_{1}^{\operatorname{loc}}([0, \infty) ; \mathcal{L}(U, Y))$, we define the (time-domain) Hankel operator $\Gamma_{h}$ associated with $h$ for $u \in \mathbf{L}_{2}^{\text {loc }}([0, \infty) ; U)$ with compact support by

$$
\left(\Gamma_{h} u\right)(t):=\int_{0}^{\infty} h(t+\tau) u(\tau) d \tau
$$

There is a nice relationship between the time-domain and frequencydomain Hankel operators.

Lemma 6.2 Suppose that $\Sigma(A, B, C, 0)$ is a stable system with impulse response $h(t)=C T(t) B$ and transfer function $\mathbf{G}$.

1. $\Gamma_{h}=\mathcal{C B}$ and it is isomorphic to $H_{\mathbf{G}}$ via

$$
\widehat{\left(\Gamma_{h} u\right)}(j \omega)=\left(H_{\mathbf{G}} \hat{u}\right)(j \omega) \text { for } u \in \mathbf{L}_{2}(0, \infty ; u) \text {. }
$$

Moreover,

$$
\left\|H_{\mathbf{G}}\right\|=\left\|\Gamma_{h}\right\|=r^{\frac{1}{2}}\left(L_{B} L_{C}\right),
$$

where $r$ denotes the spectral radius and $L_{B}, L_{C}$ are the controllability and observability Gramians, respectively, of $\Sigma(A, B, C, 0)$. 
2. If $\sigma>r^{\frac{1}{2}}\left(L_{1} L_{2}\right)$, where $L_{1}, L_{2}$ are arbitrary solutions of the Lyapunov equations (3), (4), respectively, then $N_{\sigma}:=\left(I-\frac{1}{\sigma^{2}} L_{1} L_{2}\right)^{-1} \in \mathcal{L}(Z)$.

3. $W=N_{\sigma} L_{1}$ is a bounded nonnegative solution of the following Riccati equation on $D\left(A^{*}\right)$

$$
W A_{W}^{*}+A_{W} W+\sigma^{-2} W C^{*} C W+N_{\sigma} B B^{*} N_{\sigma}^{*}=0,
$$

where $A_{W}=A-\sigma^{-2} W C^{*} C$. Moreover, $\Sigma\left(A_{W},\left[N_{\sigma} B ; W C^{*}\right], C\right)$ is a stable system and $\sigma\left(A_{W}\right) \cap \mathbb{C}_{0}^{+} \subset \sigma(A) \cap \mathbb{C}_{0}^{+}$.

Proof 1. Follows from Oostveen [28, Lemma 7.1.5].

2. Follows from [10, Lemma 2.8].

3. Follows from [10, Theorem 4.4].

Following the notation of [10] we define

$$
\mathbf{P}=\left[\begin{array}{cc}
I_{Y} & \mathbf{G} \\
0 & I_{U}
\end{array}\right], \quad J_{\sigma}=\left[\begin{array}{cc}
I_{Y} & 0 \\
0 & -\sigma^{-2} I_{U}
\end{array}\right],
$$

$\mathbf{X}$ as the transfer function of the state linear system

$$
\Sigma\left(A, \sigma^{-2} N_{\sigma}\left[L_{1} C^{*},-\sigma B\right],\left[\begin{array}{c}
C \\
B^{*} L_{2}
\end{array}\right],\left[\begin{array}{cc}
I_{Y} & 0 \\
0 & \sigma I_{U}
\end{array}\right]\right),
$$

and $\mathbf{V}$ as the transfer function of the state linear system

$$
\Sigma\left(A, \sigma^{-2}\left[-L_{1} C^{*}, B\right],\left[\begin{array}{c}
C \\
\sigma^{-1} B^{*} L_{2}
\end{array}\right] N_{\sigma},\left[\begin{array}{cc}
I_{Y} & 0 \\
0 & \sigma^{-1} I_{U}
\end{array}\right]\right) .
$$

As in [10, Lemma 6.1] we define $\mathbf{Z}$ to be transfer function of the output stable state linear system $\Sigma\left(A_{W}^{*}, L_{2} B,-\sigma^{-2} C W, 0\right)$.

Lemma 6.3 Let $\Sigma(A, B, C, 0)$ be a stable state linear system and assume that $L_{1}=L_{B}$ and $L_{2}=L_{C}$, then (34) is output stable, (35) is input stable,

$$
\mathbf{V}(s) \mathbf{X}(s)=I \text { on } \mathbb{C}_{0}^{+}
$$

and

$$
\mathbf{Z}(s)=\mathbf{V}_{21}(\bar{s})^{*} \mathbf{V}_{22}(\bar{s})^{-*} \text { on } \mathbb{C}_{0}^{+} .
$$


Proof This follows from [10, Lemma 5.3, Lemma 5.6, Lemma 5.7, Lemma $6.1]$

The following was shown in [10, Theorem 6.2].

Theorem 6.4 Let $\Sigma(A, B, C, 0)$ be a stable state linear system such that $\sigma(A) \cap i \mathbb{R}$ has measure zero, with transfer function $\mathbf{G}$ and let $L_{1}, L_{2}$ be arbitrary self-adjoint nonnegative solutions to the Lyapunov equations (3), (4), respectively. If $\sigma>r^{1 / 2}\left(L_{1} L_{2}\right)$, where $r$ denotes the spectral radius, then a solution to the suboptimal Nehari problem (28) is given by $\mathbf{K}_{c}(-s)=\mathbf{Z}(s)$ where $\mathbf{Z}$ is the transfer function of the state linear system $\Sigma\left(A_{W}^{*}, L_{2} B,-\sigma^{-2} C W, 0\right)$.

The next theorem shows that the condition $\sigma(A) \cap i \mathbb{R}$ can be replaced by the condition that $L_{1}$ and $L_{2}$ are the smallest solutions of their respective Lyapunov equations. This is an improvement of [10, Theorem 6.3] where this was proven only for $U, Y$ finite-dimensional.

Theorem 6.5 Let $\Sigma(A, B, C, 0)$ be a stable state linear system with transfer function $\mathbf{G}$ and let $L_{B}, L_{C}$ be its controllability and observability Gramian, respectively. If $\sigma>r^{1 / 2}\left(L_{B} L_{C}\right)$, where $r$ denotes the spectral radius, then a solution to the suboptimal Nehari problem (28) is given by $\mathbf{K}_{c}(-s)=\mathbf{Z}(s)$ where $\mathbf{Z}$ is the transfer function of the state linear system $\Sigma\left(A_{W}^{*}, L_{C} B,-\sigma^{-2} C W, 0\right)$.

Proof Noting that all the transfer functions $\mathbf{G}, \mathbf{V}$, and $\mathbf{Z}$ are holomorphic on $\mathbb{C}_{0}^{+}$, we perform some elementary calculations on $\mathbb{C}_{0}^{+}$. We first verify that

$$
\left[\begin{array}{c}
\mathbf{G}(s)+\mathbf{Z}(\bar{s}) \\
I
\end{array}\right]=\mathbf{P}(s) \mathbf{V}(s)^{*}\left[\begin{array}{c}
0 \\
\mathbf{V}_{22}(s)^{-*}
\end{array}\right]
$$

by expanding the right hand-side:

$$
\begin{aligned}
& {\left[\begin{array}{cc}
I & \mathbf{G}(s) \\
0 & I
\end{array}\right]\left[\begin{array}{cc}
\mathbf{V}_{11}(s)^{*} & \mathbf{V}_{21}(s)^{*} \\
\mathbf{V}_{12}(s)^{*} & \mathbf{V}_{22}(s)^{*}
\end{array}\right]\left[\begin{array}{c}
0 \\
\mathbf{V}_{22}(s)^{-*}
\end{array}\right]} \\
& =\left[\begin{array}{cc}
I & \mathbf{G}(s) \\
0 & I
\end{array}\right]\left[\begin{array}{c}
\mathbf{V}_{21}(s)^{*} \mathbf{V}_{22}(s)^{-*} \\
I
\end{array}\right]=\left[\begin{array}{c}
\mathbf{G}(s)+\mathbf{Z}(\bar{s}) \\
I
\end{array}\right],
\end{aligned}
$$


where we have used (37). We now use (38) to obtain

$$
\begin{aligned}
& (\mathbf{G}(s)+\mathbf{Z}(\bar{s}))^{*}(\mathbf{G}(s)+\mathbf{Z}(\bar{s}))-\sigma^{2} I \\
& \quad=\left[\begin{array}{c}
\mathbf{G}(s)+\mathbf{Z}(\bar{s}) \\
I
\end{array}\right]^{*} J_{\sigma}\left[\begin{array}{c}
\mathbf{G}(s)+\mathbf{Z}(\bar{s}) \\
I
\end{array}\right] \\
& \quad=\left[\begin{array}{c}
0 \\
\mathbf{V}_{22}(s)^{-*}
\end{array}\right]^{*} \mathbf{V}(s) \mathbf{P}(s)^{*} J_{\sigma} \mathbf{P}(s) \mathbf{V}(s)^{*}\left[\begin{array}{c}
0 \\
\mathbf{V}_{22}(s)^{-*}
\end{array}\right] .
\end{aligned}
$$

In $[10$, Lemma 5.1] the following $J$-spectral factorization identity is shown to hold on $\rho(A)$ for the corresponding characteristic functions

$$
\mathbf{P}(s)^{*} J_{\sigma} \mathbf{P}(s)-\mathbf{X}(s) J_{1} \mathbf{X}(s)^{*}=\mathbf{R}(s),
$$

where the remainder characteristic function is nonpositive. As in the proof of [10, Lemma 5.4], using the properties of real-analytic functions, this identity extends to the transfer functions on $\mathbb{C}_{0}^{+}$. Moreover, the remainder transfer function $\mathbf{R}(s)$ is nonpositive on $\mathbb{C}_{0}^{+}$. From (40) and (36), we obtain

$$
\mathbf{V}(s) \mathbf{P}(s)^{*} J_{\sigma} \mathbf{P}(s) \mathbf{V}(s)^{*}=J_{1}+\mathbf{V}(s) \mathbf{R}(s) \mathbf{V}(s)^{*}
$$

We now substitute this expression in (39) to calculate

$$
\begin{aligned}
& (\mathbf{G}(s)+\mathbf{Z}(\bar{s}))^{*}(\mathbf{G}(s)+\mathbf{Z}(\bar{s}))-\sigma^{2} I \\
= & -\mathbf{V}_{22}(s)^{-1} \mathbf{V}_{22}(s)^{-*}+\left[\begin{array}{c}
\mathbf{V}_{21}(s)^{*} \mathbf{V}_{22}(s)^{-*} \\
I
\end{array}\right]^{*} \mathbf{R}(s)\left[\begin{array}{c}
\mathbf{V}_{21}(s)^{*} \mathbf{V}_{22}(s)^{-*} \\
I
\end{array}\right] . \\
\leq & 0 \text { on } \mathbb{C}_{0}^{+},
\end{aligned}
$$

since $\mathbf{R}(s) \leq 0$ on $\mathbb{C}_{0}^{+}$. This shows that the holomomorphic function $\mathbf{Z}(s)$ is bounded in norm on $\mathbb{C}_{0}^{+}$and so it is in $\mathbf{H}_{\infty}$. Now $\mathbf{K}_{c}(s):=\mathbf{Z}(-s)$ is in $\mathbf{H}_{\infty}\left(\mathbb{C}_{0}^{-} ; \mathcal{L}(U, Y)\right)$ and $\mathbf{K}_{c}$ has a boundary function with $\mathbf{K}_{c}(i \omega)=\mathbf{Z}(-i \omega)$. So the function $\mathbf{K}_{c} \in \mathbf{H}_{\infty}\left(\mathbb{C}_{0}^{-} ; \mathcal{L}(U, Y)\right)$ satisfies the following for almost all $\omega \in \mathbb{R}$

$$
\|\mathbf{G}(i \omega)+\mathbf{K}(i \omega)\| \leq \sigma
$$




\section{Normalized coprime factorizations and Be- zout factors}

In this section we show that our candidate coprime factorizations are indeed coprime and we give explicit formulas for Bezout factors. Our first result is the following easy to prove but important theorem.

Theorem 7.1 Let $\mathbf{G} \in \mathbf{H}_{\infty}(\mathcal{L}(U, Y))$ be an inner function with Hankel norm strictly less than 1. Then $\mathbf{G}$ has a left inverse.

Proof From the Nehari theorem we obtain the existence of a $\mathbf{K} \in \mathbf{H}_{\infty}(\mathcal{L}(Y, U))$ such that

$$
\left\|\mathbf{G}^{*}+\mathbf{K}\right\|_{\infty}<1
$$

and using that $\mathbf{G}$ is inner we obtain

$$
\|I+\mathbf{K G}\|_{\infty} \leq\left\|\mathbf{G}^{*}+\mathbf{K}\right\|_{\infty}\|\mathbf{G}\|_{\infty}<1
$$

Since $\mathbf{K G} \in \mathbf{H}_{\infty}(\mathcal{L}(U))$ and $\mathbf{H}_{\infty}(\mathcal{L}(U))$ is a Banach algebra we obtain that $\mathbf{K G}$ is invertible over $\mathbf{H}_{\infty}(\mathcal{L}(U))$. Thus there exists a $\mathbf{Q}$ such that $\mathbf{Q K G}=I$. But then QK is a left-inverse of $\mathbf{G}$.

For us the following corollary of this theorem is of interest.

Corollary 7.2 Let $\mathbf{G}$ be a transfer function with a normalized right factorization $[\mathbf{N} ; \mathbf{M}]$. Then it is coprime if and only if the Hankel norm of $[\mathbf{N} ; \mathbf{M}]$ is strictly less than 1.

Proof The if part follows from Theorem 7.1. The only if part was shown in Oostveen and Curtain [27].

We can now state one of our main results.

Theorem 7.3 If the state linear system $\Sigma(A, B, C, D)$ is input and output stabilizable, then its transfer function has a normalized right-coprime factorization given by $\left[\mathbf{N}^{\mathrm{opt}} ; \mathbf{M}^{\mathrm{opt}}\right]$, the transfer function of the optimal right factor system (16). The transfer function $\left[\mathbf{N}^{Q} ; \mathbf{M}^{Q}\right]$ of an arbitrary right factor system (13) is also a right-coprime factorization. If $\sigma(A) \cap i \mathbb{R}$ has measure zero, then it is also normalized. 
Proof From Theorems 4.8 and 5.7 and Lemma 6.2 part 1. we see that $\left[\mathbf{M}^{\text {opt }} ; \mathbf{N}^{\text {opt }}\right]$ satisfies the assumptions of Corollary 7.2 . So $\left[\mathbf{M}^{\text {opt }} ; \mathbf{N}^{\text {opt }}\right]$ is a normalized right-coprime factorization. By Lemma $5.9\left[\mathbf{M}^{Q} ; \mathbf{N}^{Q}\right]$ is also a right-coprime factorization. The last statement folows from Lemma 5.7.

This theorem has an obvious dual and so we deduce the existence of a left and a right coprime factorization for an input and output stabilizable system. As is well-known this implies the existence of a doubly coprime factorization for an input and output stabilizable system. However, in the Youla-Bongiorno parameterization of all stabilizing controllers one needs explicit formulas for the Bezout factors. The proof of Theorem 7.1 shows that to find the Bezout factors for a right-coprime factorization we need to solve the suboptimal Nehari problem

$$
\left\|\left[-\mathbf{N}^{Q^{*}}+\mathbf{U}, \mathbf{M}^{Q^{*}}+\mathbf{V}\right]\right\|_{\infty}<1
$$

whenever $\left[\mathbf{N}^{Q} ; \mathbf{M}^{Q}\right]$ is normalized. We remark that this connection was first exploited in [13] to solve the problem of robust stabilization of normalized coprime factor descriptions. For the case $D=0$ the above suboptimal Nehari problem is equivalent to the suboptimal Nehari problem for $-\left[C ; B^{*} Q\right](s I-$ $\left.A_{Q}\right)^{-1} B$ :

$$
\left\|-\left[C, B^{*} Q\right]\left(s I-A_{Q}\right)^{-1} B+\mathbf{K}\right\|_{\infty}<1,
$$

where $\mathbf{K}(-s)=\left[\mathbf{U}(\bar{s})^{*}, \mathbf{V}(\bar{s})^{*}+I\right]$. Recall that we can only solve suboptimal Nehari problems of the form

$$
\left\|-\left[C, B^{*} Q\right]\left(s I-A_{Q}\right)^{-1} B+\mathbf{K}\right\|_{\infty} \leq \sigma
$$

where $\sigma$ is larger than the norm of the Hankel operator. For $1>\sigma>$ $r^{1 / 2}\left(P Q(I+P Q)^{-1}\right)$ this can be done and we obtain the following rather surprizing result.

Theorem 7.4 Suppose that the state linear system $\Sigma(A, B, C, 0)$ is input and output stabilizable, and let $P_{0}, Q_{0}$ denote bounded nonnegative solutions of the respective Riccati equations (14) and (12) with $D=0$. Let $\left[\mathbf{N}_{0}^{Q} ; \mathbf{M}_{0}^{Q}\right]$ and $\left[\tilde{\mathbf{N}}_{0}^{Q}, \tilde{\mathbf{M}}_{0}^{Q}\right]$ denote the transfer functions of the right and left factor systems (16) and (17) with $P_{0}, Q_{0}$ and $D=0$. Let $\sigma$ be any number satisfying $1>$ $\sigma>r^{1 / 2}\left(P_{0} Q_{0}\left(I+P_{0} Q_{0}\right)^{-1}\right.$. 
1. If either $\sigma(A) \cap i \mathbb{R}$ has measure zero or $Q_{0}$ is the smallest nonnegative solution to the control Riccati equation, then $\left[\mathbf{N}_{0}^{Q} ; \mathbf{M}_{0}^{Q}\right]$ is a normalized right-coprime factorization of $C(s I-A)^{-1} B$ with Bezout factors $\left[\tilde{\mathbf{X}}_{0}, \tilde{\mathbf{Y}}_{0}\right]$, the transfer function of the stable state linear system

$$
\Sigma\left(\tilde{A}_{L_{0}},\left[B, \tilde{L}_{0}\right], B^{*} Q_{0},[I, 0]\right),
$$

where $\tilde{W}_{0}=\left(I+P_{0} Q_{0}-\sigma^{-2} P_{0} Q_{0}\right)^{-1}, \tilde{L}_{0}=-\sigma^{-2} \tilde{W}_{0} P_{0} C^{*}$ and $\tilde{A}_{L_{0}}=$ $A+\tilde{L}_{0} C$.

2. If either $\sigma(A) \cap i \mathbb{R}$ has measure zero or $P_{0}$ is the smallest nonnegative solution to the filter Riccati equation, then the transfer function $\left[\tilde{\mathbf{N}}_{0}^{P}, \tilde{\mathbf{M}}_{0}^{P}\right]$ is a normalized left-coprime factorization of $C(s I-A)^{-1} B$ with Bezout factors $\left[\mathbf{X}_{0} ; \mathbf{Y}_{0}\right]$, the transfer function of the stable state linear system

$$
\Sigma\left(\tilde{A}_{F_{0}}, P_{0} C^{*},\left[C ; \tilde{F}_{0}\right],[I, 0]\right),
$$

where $\tilde{F}_{0}=-\sigma^{-2} B^{*} Q_{0} \tilde{W}_{0}$ and $\tilde{A}_{F_{0}}=A+B \tilde{F}_{0}$.

3. Under the above assumptions, we obtain a normalized doubly coprime factorization, i.e., $\mathbf{M}_{0}, \mathbf{N}_{0}, \tilde{\mathbf{M}}_{0}, \tilde{\mathbf{M}}_{0}, \mathbf{X}_{0}, \mathbf{Y}_{0}, \tilde{\mathbf{X}}_{0}, \tilde{\mathbf{Y}}_{0}$ satisfy (23).

Proof The statements about normalized coprime factors already follow from Theorem 7.3. As remarked above, to find the Bezout factors for a normalized right-coprime factor we need to solve the suboptimal Nehari problem for the right factor system $\Sigma\left(A_{Q_{0}}, B,\left[C ; B^{*} Q_{0}\right], 0\right)$

$$
\left\|\left[C, B^{*} Q_{0}\right]\left(s I-A_{Q_{0}}\right)^{-1} B-\mathbf{K}\right\|_{\infty} \leq \sigma .
$$

Under our assumptions we can apply either Theorem 6.5 or Theorem 6.4 to calculate a solution. Denote $\tilde{W}_{0}=\left(I+P_{0} Q_{0}-\sigma^{-2} P_{0} Q_{0}\right)^{-1}$ and $N_{\sigma}^{0}=$ $\left(I-\sigma^{-2} P_{0} Q_{0}\left(I+P_{0} Q_{0}\right)^{-1}\right)^{-1}=\tilde{W}_{0}\left(I+P_{0} Q_{0}\right)$. Then $W_{0}:=N_{\sigma}^{0}(I+$ $\left.P_{0} Q_{0}\right)^{-1} P_{0}=\tilde{W}_{0} P_{0}$ satisfies the Riccati equation given by (33) for the right factor system with $A_{W}^{Q_{0}}=A_{Q_{0}}-\sigma^{-2} \tilde{W}_{0} P_{0} C^{*} C-\sigma^{-2} \tilde{W}_{0} P_{0} Q_{0} B B^{*} Q_{0}$ and $\Sigma\left(A_{W}^{Q_{0}},\left[N_{\sigma}^{Q_{0}} B ; \tilde{W}_{0} P_{0} C^{*} ; \tilde{W}_{0} P_{0} Q_{0} B\right],\left[C, B^{*} Q_{0}\right], 0\right)$ is a stable system. From this we deduce the important fact that $\Sigma\left(A_{W}^{Q_{0}},\left[N_{\sigma}^{0} B ; \tilde{L}_{0} ; B\right],\left[C, B^{*} Q_{0}\right], 0\right)$ is a stable system.

As in Theorem 6.5 for $s \in \rho\left(A^{*}\right) \cap \mathbb{C}_{0}^{+}$we obtain

$$
\mathbf{K}_{c}(-s)=\mathbf{Z}(s)=\sigma^{-2}\left[C, B^{*} Q_{0}\right] W_{0}\left(s I-A_{W}^{Q_{0}{ }^{*}}\right)^{-1} Q_{0} B,
$$


where we have used that $\sigma\left(A_{W}^{Q_{0}}\right) \cap \mathbb{C}_{0}^{+} \subset \sigma\left(A^{Q_{0}}\right) \cap \mathbb{C}_{0}^{+} \subset \sigma(A) \cap \mathbb{C}_{0}^{+}$by Lemma 4.6 part 2 and Lemma 6.2 part 3. But $[\mathbf{U}(s), \mathbf{V}(s)+I]=\mathbf{K}_{c}(-\bar{s})^{*}$ and so for $s \in \rho(A) \cap \mathbb{C}_{0}^{+}$we have

$$
\begin{aligned}
& \mathbf{U}(s)=\sigma^{-2} B^{*} Q_{0}\left(s-A_{W}^{Q_{0}}\right)^{-1} \tilde{W}_{0} P_{0} C^{*} \\
& \mathbf{V}(s)=-I+\sigma^{-2} B^{*} Q_{0}\left(s-A_{W}^{Q_{0}}\right)^{-1} \tilde{W}_{0} P_{0} Q_{0} B
\end{aligned}
$$

From the proof of Theorem 7.1 we know that $\mathbf{U N}_{0}^{Q}-\mathbf{V M}_{0}^{Q}$ has an inverse in $\mathbf{H}_{\infty}$ and Bezout factors are provided by $\tilde{\mathbf{X}}_{0}=\left(\mathbf{V M}_{0}^{Q}-\mathbf{U N}_{0}^{Q}\right)^{-1} \mathbf{V} \in$ $\mathbf{H}_{\infty}(\mathcal{L}(U))$ and $\tilde{\mathbf{Y}}_{0}=\left(\mathbf{V M}_{0}^{Q}-\mathbf{U N}_{0}^{Q}\right)^{-1} \mathbf{U} \in \mathbf{H}_{\infty}(\mathcal{L}(Y, U))$.

We can compute these explicitly for $s \in \rho(A) \cap \rho\left(\tilde{A}_{L_{0}}\right) \cap \mathbb{C}_{0}^{+}$as follows

$$
\begin{aligned}
{\left[\mathbf{U}(s) \mathbf{N}_{0}^{Q}(s)-\mathbf{V}(s) \mathbf{M}_{0}^{Q}(s)\right]^{-1} } & =\left[I-B^{*} Q_{0}\left(s I-A_{W}^{Q_{0}}\right)^{-1} N_{\sigma}^{Q_{0}} B\right]^{-1} \\
& =I+\sigma^{-2} B^{*} Q_{0}\left(s I-\tilde{A}_{L_{0}}\right)^{-1} N_{\sigma}^{0} B
\end{aligned}
$$

where $\tilde{A}_{L_{0}}=A-\sigma^{-2} \tilde{W}_{0} P_{0} C^{*} C=A+\tilde{L}_{0} C$ as above. Using this, a simple calculation yields the formulas, for $s \in \rho(A) \cap \rho\left(\tilde{A}_{L_{0}}\right) \cap \mathbb{C}_{0}^{+}$

$$
\left[\tilde{\mathbf{X}}_{0}(s), \tilde{\mathbf{Y}}_{0}(s)\right]=[I, 0]+B^{*} Q_{0}\left(s I-\tilde{A}_{L_{0}}\right)^{-1}\left[B, \tilde{L}_{0}\right] .
$$

To identify $\left[\tilde{\mathbf{X}}_{0}, \tilde{\mathbf{Y}}_{0}\right]$ as the transfer function of the system $\Sigma_{L_{0}}=\Sigma\left(\tilde{A}_{L_{0}},\left[B, \tilde{L}_{0}\right], B^{*} Q_{0},[I, 0]\right)$, we need to show that it is either input or output stable. We show that it is both. Note that $\tilde{A}_{L_{0}}=A_{W}^{Q_{0}}-\tilde{W}_{0}(I+$ $\left.P_{0} Q_{0}\right) B B^{*} Q_{0}=A_{W}^{Q_{0}}-N_{\sigma}^{Q_{0}} B B^{*} Q_{0}$ and so

$$
\left(s I-\tilde{A}_{L_{0}}\right)^{-1}=\left(s I-A_{W}^{Q_{0}}\right)^{-1}-\left(s I-A_{W}^{Q_{0}}\right)^{-1} N_{\sigma}^{Q_{0}} B B^{*} Q_{0}\left(s I-\tilde{A}_{L_{0}}\right)^{-1} .
$$

Now we showed above that $\Sigma\left(A_{W}^{Q_{0}},\left[N_{\sigma}^{0} B ; \tilde{L}_{0}, B\right],\left[C, B^{*} Q_{0}\right], 0\right)$ is a stable system and $B^{*} Q_{0}\left(s I-\tilde{A}_{L_{0}}\right)^{-1}\left[B, \tilde{L}_{0}\right]$ is in $\mathbf{H}_{\infty}$ which implies the input stability of $\Sigma_{L_{0}}$.

The output stability follows by a similar argument, noting that $\tilde{A}_{L_{0}}=$ $A_{Q_{0}}+B B^{*} Q_{0}+\tilde{L}_{0} C$ and so

$$
\left(s I-\tilde{A}_{L_{0}}\right)^{-1}=\left(s I-A_{Q_{0}}\right)^{-1}+\left(s I-\tilde{A}_{L_{0}}\right)^{-1}\left(B B^{*} Q_{0}+\tilde{L}_{0} C\right)\left(s I-A_{Q_{0}}\right)^{-1} .
$$

A dual argument establishes part 2 .

To prove the doubly coprime property one needs to verify that

$$
\tilde{\mathbf{X}}_{0}(s) \mathbf{Y}_{0}(s)-\tilde{\mathbf{Y}}_{0}(s) \mathbf{X}_{0}(s)=0 \text { on } \mathbb{C}_{0}^{+} .
$$


A straightforward algebraic calculation shows that this indeed holds on $\mathbb{C}_{0}^{+} \cap$ $\rho(A) \cap \rho\left(\tilde{A}_{L_{0}}\right) \cap \rho\left(\tilde{A}_{F_{0}}\right)$ and it extends to $\mathbb{C}_{0}^{+}$by the analyticity of the terms.

If we can take $\sigma=1$ in the Bezout formulas, we would obtain the usual finite-dimensional formulas. This will be the case if $A_{Q}$ generates an exponentially stable semigroup, but in general this remains an open question.

For the case of non zero $D$, as in the finite-dimensional case, it is straightforward to deduce the left- and right-coprime factorizations and Bezout factors from the case $D=0$.

$$
\begin{aligned}
{\left[\begin{array}{l}
\mathbf{M}^{Q} \\
\mathbf{N}^{Q}
\end{array}\right] } & =\left[\begin{array}{ll}
I & 0 \\
D & I
\end{array}\right]\left[\begin{array}{l}
\mathbf{M}_{0}^{Q} \\
\mathbf{N}_{0}^{Q}
\end{array}\right] \\
{\left[\begin{array}{c}
\mathbf{X} \\
\mathbf{Y}
\end{array}\right] } & =\left[\begin{array}{cc}
I & D \\
0 & I
\end{array}\right]\left[\begin{array}{l}
\mathbf{X}_{0} \\
\mathbf{Y}_{0}
\end{array}\right] \\
{\left[\begin{array}{ll}
\tilde{\mathbf{M}}^{P} & \tilde{\mathbf{N}}^{P}
\end{array}\right] } & =\left[\begin{array}{ll}
\tilde{\mathbf{M}}_{0}^{P} & \tilde{\mathbf{N}}_{0}^{P}
\end{array}\right]\left[\begin{array}{cc}
I & D \\
0 & I
\end{array}\right], \\
{\left[\begin{array}{ll}
\tilde{\mathbf{X}} & \tilde{\mathbf{Y}}
\end{array}\right] } & =\left[\begin{array}{ll}
\tilde{\mathbf{X}}_{0} & \tilde{\mathbf{Y}}_{0}
\end{array}\right]\left[\begin{array}{cc}
I & 0 \\
D & I
\end{array}\right] .
\end{aligned}
$$

It is easy to see that this provides a doubly coprime factorization, but that it is not normalized. In the next theorem we obtain formulas for a normalized doubly coprime factorization for the $D \neq 0$ case.

Theorem 7.5 Suppose that the state linear system $\Sigma(A, B, C, D)$ is input and output stabilizable, $\sigma$ is any number satisfying $1>\sigma>r^{1 / 2}(P Q(I+$ $P Q)^{-1}$.

1. If either $\sigma(A) \cap i \mathbb{R}$ has measure zero or $Q=Q^{\mathrm{opt}}$, the smallest nonnegative solution to the control Riccati equation, then $\left[\mathbf{N}^{Q} ; \mathbf{M}^{Q}\right]$ is a normalized right-coprime factorization of $\mathbf{G}$ with Bezout factors $[\tilde{\mathbf{X}}, \tilde{\mathbf{Y}}]$, the transfer function of the stable state linear system

$$
\Sigma\left(\tilde{A}_{L},[B+\tilde{L} D, \tilde{L}], S^{-1 / 2} B^{*} Q, S^{-1 / 2}\left[I,-D^{*}\right]\right),
$$

where $\tilde{W}=\left(I+P Q-\sigma^{-2} P Q\right)^{-1}, \tilde{L}=-\left(\sigma^{-2} \tilde{W} P C^{*}+B D^{*}\right) R^{-1}$ and $\tilde{A}_{L}=A+\tilde{L} C$.

2. If either $\sigma(A) \cap i \mathbb{R}$ has measure zero or $P=P^{\mathrm{opt}}$, the smallest nonnegative solution to the filter Riccati equation, then the transfer function 
$\left[\tilde{\mathbf{N}}^{P}, \tilde{\mathbf{M}}^{P}\right]$ is a normalized left-coprime factorization of $\mathbf{G}$ with Bezout factors $[\mathbf{X} ; \mathbf{Y}]$, the transfer function of the stable state linear system

$$
\Sigma\left(\tilde{A}_{F}, P C^{*} R^{-1 / 2},[C+D \tilde{F} ; \tilde{F}],\left[I,-D^{*}\right] R^{-1 / 2}\right),
$$

where $\tilde{F}=-S^{-1}\left(\sigma^{-2} B^{*} Q \tilde{W}+D^{*} C\right)$ and $\tilde{A}_{F}=A+B \tilde{F}$.

3. Under the above assumptions, we obtain a normalized doubly coprime factorization, i.e., $\mathbf{M}, \mathbf{N}, \tilde{\mathbf{M}}, \tilde{\mathbf{M}}, \mathbf{X}, \mathbf{Y}, \tilde{\mathbf{X}}, \tilde{\mathbf{Y}}$ satisfy (23).

Proof First we note that $\Sigma_{1}=\Sigma\left(A-B S^{-1} D^{*} C, B S^{-1 / 2}, R^{-1 / 2} C, 0\right)$ has the same control and filter Riccati equations as $\Sigma(A, B, C, D)$. We apply Theorem 7.4 to system $\Sigma_{1}$ and obtain the normalized right-coprime factors defined on $\rho\left(A_{Q}\right) \cap \mathbb{C}_{0}^{+}$by

$$
\left[\mathbf{N}_{1}(s) ; \mathbf{M}_{1}(s)\right]=[0 ; I]+\left[R^{-1 / 2} C ;-S^{-1 / 2} B^{*} Q\right]\left(s I-A_{Q}\right)^{-1} B S^{-1 / 2}
$$

and the Bezout factors defined on $\rho\left(\tilde{A}_{L}\right) \cap \mathbb{C}_{0}^{+}$by

$$
\left[\tilde{\mathbf{X}}_{1}(s), \tilde{\mathbf{Y}}_{1}(s)\right]=[I, 0]+S^{-1 / 2} B^{*} Q\left(s I-\tilde{A}_{L}\right)^{-1}\left[B S^{-1 / 2},-\sigma^{-2} \tilde{W} P C^{*} R^{-1 / 2}\right] .
$$

The right-coprime factors $\left[\mathbf{N}^{Q} ; \mathbf{M}^{Q}\right]$ of $\Sigma(A, B, C, D)$ satisfy

$$
\left[\begin{array}{l}
\mathbf{N}_{1} \\
\mathbf{M}_{1}
\end{array}\right]=\left[\begin{array}{cc}
R & 0 \\
0 & S
\end{array}\right]^{-1 / 2}\left[\begin{array}{cc}
I & -D \\
D^{*} & I
\end{array}\right]\left[\begin{array}{l}
\mathbf{N} \\
\mathbf{M}
\end{array}\right]
$$

We already know that $\tilde{\mathbf{X}}_{1} \mathbf{M}_{1}-\tilde{\mathbf{Y}}_{1} \mathbf{N}_{1}=I$ and so can deduce that Bezout factors for $\left[\mathbf{N}^{Q} ; \mathbf{M}^{Q}\right]$ are given by

$$
\left[\begin{array}{cc}
\tilde{\mathbf{X}} & \tilde{\mathbf{Y}}
\end{array}\right]=\left[\begin{array}{ll}
\tilde{\mathbf{X}}_{1} & \tilde{\mathbf{Y}}_{1}
\end{array}\right]\left[\begin{array}{cc}
S & 0 \\
0 & R
\end{array}\right]^{-1 / 2}\left[\begin{array}{cc}
I & -D^{*} \\
D & I
\end{array}\right]
$$

Dual arguments show that the normalized left-coprime factors of $\Sigma_{1}$ are given on $\rho\left(A_{P}\right) \cap \mathbb{C}_{0}^{+}$by

$$
\left[\tilde{\mathbf{N}}_{1}(s), \tilde{\mathbf{M}}_{1}(s)\right]=[0, I]+R^{-1 / 2} C\left(s I-A_{P}\right)^{-1}\left[B S^{-1 / 2},-P C^{*} R^{-1 / 2}\right]
$$

and the Bezout factors are given on $\rho\left(\tilde{A}_{F}\right) \cap \mathbb{C}_{0}^{+}$by

$$
\left[\mathbf{X}_{1}(s) ; \mathbf{Y}_{1}(s)\right]=[I, 0]+\left[R^{-1 / 2} C ;-\sigma^{-2} S^{-1 / 2} B^{*} Q \tilde{W}\right]\left(s I-\tilde{A}_{F}\right)^{-1} P C^{*} R^{-1 / 2} .
$$


As above, we can deduce the Bezout factors for $[\tilde{\mathbf{N}}, \tilde{\mathbf{M}}]$ to be given by

$$
\left[\begin{array}{c}
\mathbf{Y} \\
-\mathbf{X}
\end{array}\right]=\left[\begin{array}{cc}
I & D^{*} \\
-D & I
\end{array}\right]\left[\begin{array}{ll}
S & 0 \\
0 & R
\end{array}\right]^{-1 / 2}\left[\begin{array}{c}
\mathbf{Y}_{1} \\
-\mathbf{X}_{1}
\end{array}\right]
$$

It is easily checked that with the obtained pairs of Bezout factors we have a doubly coprime factorization, i.e. (23) holds. For example we have

$$
\begin{aligned}
& {\left[\begin{array}{ll}
\tilde{\mathbf{X}} & \tilde{\mathbf{Y}}
\end{array}\right]\left[\begin{array}{c}
\mathbf{Y} \\
-\mathbf{X}
\end{array}\right] } \\
= & {\left[\tilde{\mathbf{X}}_{1}, \tilde{\mathbf{Y}}_{1}\right]\left[\begin{array}{cc}
S & 0 \\
0 & R
\end{array}\right]^{-1 / 2}\left[\begin{array}{cc}
I & -D^{*} \\
D & I
\end{array}\right]\left[\begin{array}{cc}
I & D^{*} \\
-D & I
\end{array}\right]\left[\begin{array}{cc}
S & 0 \\
0 & R
\end{array}\right]^{-1 / 2}\left[\begin{array}{c}
\mathbf{Y}_{1} \\
-\mathbf{X}_{1}
\end{array}\right] } \\
= & \tilde{\mathbf{X}}_{1} \mathbf{Y}_{1}-\tilde{\mathbf{Y}}_{1} \mathbf{X}_{1} \\
= & 0
\end{aligned}
$$

which shows that the factorization is doubly coprime.

Even if $\left[\mathbf{N}^{Q} ; \mathbf{M}^{Q}\right]$ is not normalized, then it is a right-coprime factorization, but in general, we do not know if the candidate Bezout factors from Theorem 7.5 will be in $\mathbf{H}_{\infty}$. We can always find suitable Bezout factors using Lemma 5.9, but this leads to messy formulas.

\section{Integrated nodes and reciprocals}

First we briefy review the definition of an operator node (see Staffans [32, Section 4.7]) and introduce the concept of an integrated node (this is a specialization of the concept of a distributional resolvent linear system introduced in Opmeer [26]). $U, Y, Z$ are separable Hilbert spaces. An operator node is specified by three operators $A, B, C$ and a characteristic function $\mathfrak{G}$. These are assumed to satisfy:

- $A$ is a closed densely defined operator on $Z$ with nonempty resolvent set.

- $C \in \mathcal{L}(D(A), Y)$ is bounded where $D(A)$ is equipped with the graph norm.

- $B^{*} \in \mathcal{L}\left(D\left(A^{*}\right), U\right)$ is bounded where $D\left(A^{*}\right)$ is equipped with the graph norm.

- $\mathfrak{G}: \rho(A) \rightarrow \mathcal{L}(U, Y)$ satisfies

$$
\mathfrak{G}(s)-\mathfrak{G}(\alpha)=(\alpha-s) C(s I-A)^{-1}(\alpha I-A)^{-1} B .
$$

for $\alpha, s \in \rho(A)$. The dual of an operator node is specified by the operators $A^{*}, C^{*}, B^{*}, \mathfrak{G}(\bar{\alpha})^{*}$. 
Definition 8.1 An integrated node is an operator node for which $\rho(A)$ contains a right half-plane and there exist a polynomial $p$ such that for $s$ in this right half-plane

$$
\left\|(s I-A)^{-1}\right\| \leq p(|s|) .
$$

The above resolvent estimate is equivalent to the statement: $A$ generates an exponentially bounded integrated semigroup (see Arendt et al. [1, Section $3.2])$.

Note that $(s I-A)^{-1} B, C(s I-A)^{-1}$ and the characteristic function of an integrated node are polynomially bounded on the same right half-plane as the resolvent.

We recall that the Laplace transform can be defined for certain Banach space valued distributions and that the image of the set of Laplace transformable distributions is exactly the set of functions defined on some right half-plane that are analytic and polynomially bounded (see Schwartz [30]). This allows us to define the state and output of an integrated node as Laplace transformable distributions ( $Z$-valued and $Y$-valued, respectively).

Definition 8.2 For an initial state $z_{0} \in Z$ and a Laplace transformable distribution $u$ the state and output of an integrated node are defined through their Laplace transforms by

$$
\begin{aligned}
& \hat{z}(s)=(s I-A)^{-1} z_{0}+(s I-A)^{-1} B \hat{u}(s), \\
& \hat{y}(s)=C(s I-A)^{-1} z_{0}+\mathfrak{G}(s) \hat{u}(s),
\end{aligned}
$$

with $s$ in some right half-plane that is contained in $\rho(A)$.

The finite cost condition for integrated nodes is defined exactly the same as for state linear systems.

Definition 8.3 The finite cost condition for an integrated node is: for every $z_{0} \in Z$ there exists a $u \in \mathbf{L}_{2}(0, \infty ; U)$ such that the corresponding output $y \in \mathbf{L}_{2}(0, \infty ; Y)$.

We define stability of integrated nodes. Note that for state linear systems this definition is equivalent to Definition 2.1.

Definition 8.4 An integrated node is output stable if for every $z_{0} \in Z$ the output with zero input is in $\mathbf{L}_{2}(0, \infty ; Y)$ and satisfies

$$
\|y\|_{\mathbf{L}_{2}(0, \infty ; Y)} \leq M\left\|z_{0}\right\|
$$


for some $M>0$ independent of $z_{0}$.

An integrated node is input-output stable if for every input $u \in \mathbf{L}_{2}(0, \infty ; U)$ the output with zero initial condition is in $\mathbf{L}_{2}(0, \infty ; Y)$ and satisfies

$$
\|y\|_{\mathbf{L}_{2}(0, \infty ; Y)} \leq M\|u\|_{\mathbf{L}_{2}(0, \infty ; U)}
$$

for some $M>0$ independent of $u$.

An integrated node is input stable if its dual node is output stable. An integrated node is called stable if it is input, output and input-output stable.

The transfer function of an integrated node is defined as the maximal analytic extension of a restriction of the characteristic function. The precise defintion is as follows.

Definition 8.5 Consider an integrated node specified by the operators $A, B, C$ and the characteristic function $\mathfrak{G}$. Let $\omega$ be such that $\mathbb{C}_{\omega}^{+} \subset \rho(A)$. Define $\mathbf{G}_{1}: \mathbb{C}_{\omega}^{+} \rightarrow \mathcal{L}(U, Y)$ as the restriction of the characteristic function. Let $\gamma \in[-\infty, \omega]$ be such that $\mathbf{G}_{1}$ has an analytic extension to $\mathbb{C}_{\gamma}^{+}$, but not to $\mathbb{C}_{\sigma}^{+}$for any $\sigma<\gamma$. Define the transfer function $\mathbf{G}: \mathbb{C}_{\gamma}^{+} \rightarrow \mathcal{L}(U, Y)$ as the analytic extension of $\mathbf{G}_{1}$.

We note that as in the case of state linear systems we have $\mathfrak{G}(s)=\mathbf{G}(s)$ for $s$ in a right half-plane as long as this right half-plane is contained in $\rho(A)$. However, as in the case of state linear systems, these equalities may not hold outside such a right half-plane.

The concept of a reciprocal system was introduced in [8] for well-posed linear system and it easily extends to operator nodes.

Definition 8.6 Suppose that the operator node $\Sigma$ with generating operators $A, B, C$ and characteristic function $\mathfrak{G}$ is such that $0 \in \rho(A)$. Its reciprocal system is the state linear system $\Sigma\left(A^{-1}, A^{-1} B,-C A^{-1}, \mathfrak{G}(0)\right)$.

The following theorem follows as in [8].

Theorem 8.7 Suppose that $A, B, C$ are the generating operators of an integrated node $\Sigma$ with transfer function $\mathbf{G}$ and zero is in the resolvent set of A. Denote the characteristic function of its reciprocal system by $\mathfrak{G}_{r}$ and the transfer function of its reciprocal system by $\mathbf{G}_{r}$. Then

1. $\mathfrak{G}(s)=\mathfrak{G}_{r}\left(\frac{1}{s}\right)$ whenever $s$ is in the resolvent set of $A$. 
2. $\Sigma$ is output stable if and only if its reciprocal system is output stable.

3. $\Sigma$ is input stable if and only if its reciprocal system is input stable.

4. $\Sigma$ is a stable system if and only if its reciprocal system is a stable system. In this case, we have $\mathbf{G}(s)=\mathbf{G}_{r}\left(\frac{1}{s}\right)$ for $s \in \mathbb{C}_{0}^{+}$.

Although the following theorem was stated in [25] for well-posed linear systems, its proof applies to integrated nodes as well.

Theorem 8.8 An integrated node $\Sigma$ with $0 \in \rho(A)$ satisfies the finite cost condition if and only if its reciprocal system does. Moreover, if the finite cost condition is satisfied, then there exist unique optimal controls for $\Sigma$ and for its reciprocal system and the optimal costs are equal.

Since the reciprocal system is a state linear system, the results stated earlier in this article are applicable to it. First we consider the regulator problem and apply Theorem 4.4 to the reciprocal system. We obtain the optimal cost operator for this problem as the smallest bounded nonnegative solution to the following reciprocal control Riccati equation.

$$
\begin{aligned}
& A^{-*} Q+Q A^{-1}+A^{-*} C^{*} C A^{-1}= \\
& \quad\left(Q A^{-1} B-A^{-*} C^{*} D_{r}\right) S_{r}^{-1}\left(B^{*} A^{-*} Q-D_{r}^{*} C A^{-1}\right),
\end{aligned}
$$

where $D_{r}=\mathfrak{G}(0)$ and $S_{r}=I+D_{r}^{*} D_{r}$. According to Theorem 8.8, the optimal cost operator for the regulator problem for an integrated node is the smallest bounded nonnegative solution to the above reciprocal control Riccati equation.

Next we consider the problem of coprime factorizations for an integrated node.

Theorem 8.9 Let $\Sigma$ be an integrated node with $0 \in \rho(A)$. Assume that the finite cost condition for $\Sigma$ and for its dual system are both satisfied. Then the transfer function of $\Sigma$ has a normalized doubly coprime factorization.

Proof It follows from Theorem 8.8 that the reciprocal system $\Sigma_{r}$ of $\Sigma$ and its dual satisfy the finite cost condition. In other words, $\Sigma_{r}$ is input and output stabilizable. So we can apply Theorem 7.5 to the reciprocal system $\Sigma_{r}$ to show that its transfer function has a normalized doubly coprime factorization. Denote the coprime factors and Bezout factors by $\mathbf{M}_{r}, \mathbf{N}_{r}, \tilde{\mathbf{M}}_{r}, \tilde{\mathbf{M}}_{r}, \mathbf{X}_{r}, \mathbf{Y}_{r}$, $\tilde{\mathbf{X}}_{r}, \tilde{\mathbf{Y}}_{r}$ and define $\mathbf{M}(s)=\mathbf{M}_{r}(1 / s), \mathbf{N}(s)=\mathbf{N}_{r}(1 / s), \tilde{\mathbf{M}}(s)=\tilde{\mathbf{M}}_{r}(1 / s)$, 
$\tilde{\mathbf{M}}(s)=\tilde{\mathbf{M}}_{r}(1 / s), \mathbf{X}(s)=\mathbf{X}_{r}(1 / s), \mathbf{Y}(s)=\mathbf{Y}_{r}(1 / s), \tilde{\mathbf{X}}(s)=\tilde{\mathbf{X}}_{r}(1 / s)$, $\tilde{\mathbf{Y}}(s)=\tilde{\mathbf{Y}}_{r}(1 / s)$. Then it is easily seen that this provides a normalized doubly coprime factorization of the transfer function of $\Sigma$. As an example of the arguments involved we show that the transfer function of $\Sigma$ equals $\mathrm{NM}^{-1}$ on some right half-plane.

By Theorem 5.6 part 4 . and Lemma 4.6 part 2. we see that $\mathfrak{G}_{r}(s)=$ $\mathfrak{N}_{r}(s) \mathfrak{M}_{r}(s)^{-1}$ for $s \in \mathbb{C}_{0}^{+} \cap \rho\left(A_{r}\right)$. Since $s \in \mathbb{C}_{0}^{+} \cap \rho(A)$ if and only if $1 / s \in \mathbb{C}_{0}^{+} \cap \rho\left(A_{r}\right)$ this gives $\mathfrak{G}(s)=\mathfrak{N}(s) \mathfrak{M}(s)^{-1}$ for $s \in \mathbb{C}_{0}^{+} \cap \rho(A)$. Using that $\mathfrak{N}=\mathbf{N}, \mathfrak{M}=\mathbf{M}$ and $\mathfrak{G}=\mathbf{G}$ on some right half-plane we obtain $\mathbf{G}(s)=\mathbf{N}(s) \mathbf{M}(s)^{-1}$ for $s$ in some right half-plane.

Remark 8.10 From the proof of Theorem 8.9 and Theorem 7.5 one can deduce explicit formulas for the coprime factors and Bezout factors. These are in terms of the generating operators of the reciprocal system. This leads to a complete Youla-Bongiorno type parameterization of all stabilizing controllers as in [5].

Remark 8.11 It is possible to obtain the existence of a normalized doubly coprime factorization without the assumption $0 \in \rho(A)$. One should reprove all our results on state linear systems for discrete-time systems and then use the connection between the linear quadratic regulator problem for integrated nodes and that for it Cayley transform (a discrete-time system) as was shown in [25] (for well-posed linear systems, but the proof applies to integrated nodes as well). Finally, we remark that the results of this section can be extended to the slightly more general case of distributional resolvent linear systems as introduced in [26].

\section{References}

[1] Wolfgang Arendt, Charles J. K. Batty, Matthias Hieber, and Frank Neubrander. Vector-valued Laplace transforms and Cauchy problems, volume 96 of Monographs in Mathematics. Birkhäuser Verlag, Basel, 2001.

[2] R.F. Curtain and G. Weiss and M. Weiss. Coprime Factorizations for Regular Linear Systems, Automatica, 32: 1519-1532, 1996. 
[3] R.F. Curtain and J.C. Oostveen. Necessary and sufficient conditions for strong stability of distributed parameter systems, Systems and Control Letters, 37: 11-18, 1999.

[4] R.F. Curtain and J.C. Oostveen. Normalized coprime factorizations for strongly stabilizable systems. In Advances in Mathematical Control Theory (in honour of Diederich Hinrichsen), pages 265-280, Boston, 2000. Birkhäuser.

[5] Ruth F. Curtain, George Weiss and Martin Weiss, Stabilization of irrational transfer functions with internal loop, p. 179-208 in Systems, Approximation, Singular integral operators and related topics, Int. workshop on operator theory and applications, IWOTA 2000. Eds., Alexander A. Borichev and Nikolai K. Nikolski, Vol. 129, 2001, in series Advances in operator Theory and Applications, Birkhäuser.

[6] R.F. Curtain and H.J. Zwart. An Introduction to Infinite-Dimensional Linear Systems Theory. Springer-Verlag, New York, 1995.

[7] R.F. Curtain and A.J. Sasane. Compactness and nuclearity of the Hankel operator and internal stability of infinite-dimensional state linear systems. Int. J. Control, 48: 407-414, 2003.

[8] R.F. Curtain. Regular linear systems and their reciprocals: applications to Riccati equations. Systems and Control Letters, 49: 81-89, 2003.

[9] R.F. Curtain. Riccati equations for stable well-posed linear systems; the generic case. SIAM journal of Control and Optimization, 42: 1681-1702, 2003.

[10] R.F. Curtain and M.R. Opmeer. The suboptimal Nehari problem for well-posed linear systems. SIAM journal of Control and Optimization, to appear.

[11] B.A. Francis. A Course in $H_{\infty}$ Control Theory. Lecture Notes in Control and Information Sciences, Vol.88, Springer Verlag, Berlin, 1987.

[12] T.T. Georgiou and M.C. Smith. Optimal robustness in the gap metric. IEEE Trans. Automat. Control, 35: 673-686, 1990. 
[13] K. Glover and D. McFarlane. Robust stabilization of normalized coprime factor plant descriptions with $H_{\infty}$-bounded uncertainty. IEEE Trans. Autom. Control, AC-43: 821-830, 1989.

[14] P. Grabowski. On the spectral-Lyapunov approach to parametric optimization of distributed parameter systems. IMA Journal of Mathematical Control and Information, 7: 317-338, 1991.

[15] S. Hansen and G. Weiss. New results on the operator Carleson measure criterion. IMA Journal of Mathematical Control and Information, 14: 3-32, 1997.

[16] Y. Inouye. Parametrization of compensators for linear systems with transfer functions of bounded type. Proceedings of the 27th IEEE Conference on Decision and Control, 2083-2088, 1988.

[17] T. Kato. Perturbation Theory of Linear Operators. Springer Verlag, New York, 1966.

[18] A, Kheifets. Parametrization of solutions of the Nehari problem and nonorthogonal dynamics. In Operator thory and interpoloation (Bloomington, IN, 1996), volume 115 of Oper. Theory Adv. Appl. pages 213233. Birkhäuser, Basel, 2000.

[19] I. Lasiecka and R. Triggiani. Differential and algebraic Riccati equations with application to boundary/point control problems: continuous theory and approximation theory, volume 164 of Lecture Notes in Control and Information Sciences. Springer-Verlag, Berlin, 1991.

[20] Irena Lasiecka and Roberto Triggiani. Control theory for partial differential equations: continuous and approximation theories. I, volume 74 of Encyclopedia of Mathematics and its Applications. Cambridge University Press, Cambridge, 2000. Abstract parabolic systems.

[21] Irena Lasiecka and Roberto Triggiani. Control theory for partial differential equations: continuous and approximation theories. II, volume 75 of Encyclopedia of Mathematics and its Applications. Cambridge University Press, Cambridge, 2000. Abstract hyperbolic-like systems over a finite time horizon. 
[22] D.G. Meyer and G.F. Franklin. A connection between normalized coprime factorizations and linear quadratic regulator theory. IEEE Trans. Automat. Control, 32:227-228, 1987.

[23] K. Mikkola. Infinite-Dimensional Linear Systems, Optimal Control and Riccati Equations. PhD thesis, October 2002, Helsinki University of Technology. Available at http://www.math.hut.fi/reports

[24] C.N. Nett and C.A. Jacobson and M.A. Balas. A connection between state space and doubly coprime factorizations, IEEE Trans. Autom. Control 29: 831-832, 1984.

[25] Mark R. Opmeer and Ruth F. Curtain. New Riccati equations for wellposed linear systems. Systems Control Lett., 52(5):339-347, 2004.

[26] Mark R. Opmeer. Infinite-dimensional linear systems: a distributional approach. Proceedings of the London Mathematical Society, to appear.

[27] J.C. Oostveen and R.F. Curtain. Robustly stabilizing controllers for disipative infinite-dimensional systems with collocated actuators and sensors. Automatica, 36: 337-348, 2000.

[28] J.C. Oostveen. Strongly Stabilizable Distributed Parameter Systems. SIAM, Philadelphia, 2000.

[29] Vladimir V. Peller. Hankel operators and their applications, Springer Monographs in Mathematics, Springer-Verlag, New York, 2003.

[30] Laurent Schwartz. Théorie des distributions. Publications de l'Institut de Mathématique de l'Université de Strasbourg, No. IX-X. Nouvelle édition, entiérement corrigée, refondue et augmentée. Hermann, Paris, 1966 .

[31] M. C. Smith. On stabilization and the existence of coprime factorizations. IEEE Trans. Automat. Control. 34: 1005-1007, 1989.

[32] Olof Staffans. Well-posed linear systems, volume 103 of Encyclopedia of Mathematics and its Applications. Cambridge University Press, Cambridge, 2005.

[33] O.J. Staffans. Coprime factorizations and well-posed linear systems. SIAM Journal on Control and Optimization, 36:1268-1292, 1998. 
[34] George Weiss. Representation of shift-invariant operators on $L^{2}$ by $H^{\infty}$ transfer functions: an elementary proof, a generalization to $L^{p}$, and a counterexample for $L^{\infty}$. Math. Control Signals Systems, 4(2):193-203, 1991.

[35] H.J. Zwart. Transfer functions for infinite-dimensional systems. Systems and Control Letters, 52: 247-255, 2004. 